\title{
OPTIMAL CONTROL OF QUASILINEAR PARABOLIC EQUATIONS*
}

\author{
Eduardo Casas ${ }^{\dagger}$ Luis A. Fernández ${ }^{\ddagger}$ and Jiongmin Yong§
}

\section{Introduction}

There exists a vast literature concerning with the optimal control of nonlinear evolution systems, even with state constraints. By using different approaches (weak solutions, mild solutions,...) and methods (penalization, linearization, relaxation,...) different kind of first order necessary optimality conditions (of integral type or maximum principle type) are obtained: in the semilinear case, see for instance [2], [6], [9], [18], [19] and the bibliography cited in these works; with regard to the quasilinear case, there are various studies about the existence of optimal controls (among others [11], [13] and [14]) and a few others related with the derivation of the optimality system as [1], [17] and more recently [16]; see [15] for retarded systems. Nevertheless, the assumptions in these studies are so restrictive that make the results inapplicable to classical quasilinear parabolic equations. For instance, the operator

$$
y_{t}(x, t)-d i v_{x}\left(\left(k+\left|\nabla_{x} y(x, t)\right|\right)^{\alpha-2} \nabla_{x} y(x, t)\right)
$$

neither satisfies the condition ii) of Lemma 3.3 nor (C2) in [1] (and consequently the condition $H(A)_{4}-(6)$ imposed in [16], which coincides with the first one).

On the other hand, the work [17] contains a non well clarified point (the uniqueness of solution asserted in Theorem 6.2 may be not true), although the argumentation can be modified in such a way that most of the final consequences remain valid for the operator under consideration, which has a very particular structure that does not include (1.1).

This paper deals with optimal control problems of systems governed by quasilinear parabolic equations in divergence form, whose prototype is (1.1). Our aim is to prove existence of solution and derive some optimality conditions in a rigorous way.

Optimal control of systems governed by quasilinear elliptic equations has been studied in some previous authors' works (see for instance [3], [4], [5] and the bibliography cited therein), where even state constraints were considered.

The rest of the paper is organized as follows: in Section 2 we study the state equation; in Section 3 we present the control problem under consideration and derive the existence of

*The first two authors were partially supported by Dirección General de Investigación Científica y Técnica (Madrid) and the third one by the NSF of China under grant 19131050 and the Fok Ying Tung Education Foundation

${ }^{\dagger}$ Departamento de Matemática Aplicada y Ciencias de la Computación, E.T.S.I. de Caminos, Universidad de Cantabria, 39071 Santander, Spain.

†Departamento de Matemáticas, Estadística y Computación, Facultad de Ciencias, Universidad de Cantabria, 39071 Santander, Spain.

$\S$ Departament of Mathematics, Fudan University, Shanghai 200433, China. 
optimal controls; in Section 4, we study the conditions under which the functional control-state is differentiable. Finally, in Section 5, we derive the optimality systems for different types of operators.

\section{The state equation}

Let $\Omega$ be a bounded open subset of $\mathbb{R}^{n}$ with Lipschitz continuous boundary $\Gamma$ and $0<T<$ $+\infty$. Consider the parabolic initial-boundary value problem

$$
\begin{cases}y_{t}(x, t)+A y(x, t)=u(x, t) & \text { in } Q_{T}=\Omega \times(0, T) \\ y(x, t)=0 & \text { on } \Sigma=\Gamma \times(0, T) \\ y(x, 0)=y_{0}(x) & \text { in } \Omega\end{cases}
$$

where $A$ is the quasilinear differential operator

$$
\begin{gathered}
A y(x, t)=-\operatorname{div}_{x}\left(a\left(x, t, \nabla_{x} y(x, t)\right)+a_{0}(x, t, y(x, t))=\right. \\
=-\sum_{j=1}^{n} \frac{\partial}{\partial x_{j}}\left(a_{j}\left(x, t, \frac{\partial y}{\partial x_{1}}(x, t), \ldots, \frac{\partial y}{\partial x_{n}}(x, t)\right)\right)+a_{0}(x, t, y(x, t)) .
\end{gathered}
$$

On the coefficients we will assume the following conditions:

$$
\begin{gathered}
\left\{\begin{array}{l}
a_{j}(\cdot, \cdot, \eta) \text { is a measurable function in } Q_{T} \quad \forall \eta \in \mathbb{R}^{n} \\
a_{j}(x, t, \cdot) \text { belongs to } C^{1}\left(\mathbb{R}^{n}\right) \text { a.e. }(x, t) \in Q_{T} \quad j=1, \ldots, n
\end{array}\right. \\
\left\{\begin{array}{l}
a_{0}(\cdot, \cdot, y) \text { is a measurable function in } Q_{T} \quad \forall y \in \mathbb{R} \\
a_{0}(x, t, \cdot) \text { belongs to } C^{1}(\mathbb{R}) \text { a.e. }(x, t) \in Q_{T}
\end{array}\right. \\
\sum_{i, j=1}^{n} \frac{\partial a_{j}}{\partial \eta_{i}}(x, t, \eta) \xi_{i} \xi_{j} \geq \Lambda_{1}(k+|\eta|)^{\alpha-2}|\xi|^{2} \\
\sum_{i, j=1}^{n}\left|\frac{\partial a_{j}}{\partial \eta_{i}}(x, t, \eta)\right| \leq \Lambda_{2}(k+|\eta|)^{\alpha-2} \\
0 \leq \frac{\partial a_{0}}{\partial y}(x, t, y) \leq \varphi_{0}(|y|) \\
a_{0}(x, t, 0)=a_{j}(x, t, 0)=0 \quad j=1, \ldots, n
\end{gathered}
$$

for some $\alpha>\max \{2 n /(n+2), 1\}, k \geq 0, \Lambda_{1}, \Lambda_{2}>0$, some positive increasing function $\varphi_{0}$, and all $(x, t) \in Q_{T}, y \in \mathbb{R}$ and $\eta, \xi \in \mathbb{R}^{n}$.

Linear and semilinear parabolic equations are included in the case $\alpha=2$.

Some spaces appear in the study of the parabolic initial-boundary value problems like (2.1) in a natural way

$$
W^{\alpha}(0, T)=\left\{y \in L^{\alpha}\left(0, T ; W_{0}^{1, \alpha}(\Omega)\right): y_{t} \in L^{\alpha^{\prime}}\left(0, T ; W^{-1, \alpha^{\prime}}(\Omega)\right)\right\}
$$

and

$$
W^{1, \alpha^{\prime}}\left(0, T ; W^{-1, \alpha^{\prime}}(\Omega)\right)=\left\{y \in L^{\alpha^{\prime}}\left(0, T ; W^{-1, \alpha^{\prime}}(\Omega)\right): y_{t} \in L^{\alpha^{\prime}}\left(0, T ; W^{-1, \alpha^{\prime}}(\Omega)\right)\right\}
$$


with $\alpha^{\prime}=\frac{\alpha}{\alpha-1}$, that equipped respectively with the norms

$$
\|y\|_{W^{\alpha}(0, T)}=\|y\|_{L^{\alpha}\left(0, T ; W_{0}^{1, \alpha}(\Omega)\right)}+\left\|y_{t}\right\|_{L^{\alpha^{\prime}}\left(0, T ; W^{-1, \alpha^{\prime}}(\Omega)\right)}
$$

and

$$
\|y\|_{W^{1, \alpha^{\prime}}\left(0, T ; W^{-1, \alpha^{\prime}}(\Omega)\right)}=\|y\|_{L^{\alpha^{\prime}\left(0, T ; W^{-1, \alpha^{\prime}}(\Omega)\right)}}+\left\|y_{t}\right\|_{L^{\alpha^{\prime}\left(0, T ; W^{-1, \alpha^{\prime}}(\Omega)\right)}}
$$

are Banach spaces. Moreover

$$
W^{1, \alpha^{\prime}}\left(0, T ; W^{-1, \alpha^{\prime}}(\Omega)\right) \subset C\left([0, T] ; W^{-1, \alpha^{\prime}}(\Omega)\right) \quad \text { with continuous imbedding. }
$$

On the other hand, since we are assuming $\alpha>2 n /(n+2)$, by virtue of the Sobolev imbeddings it holds that

$$
W_{0}^{1, \alpha}(\Omega) \subset L^{2}(\Omega) \subset W^{-1, \alpha^{\prime}}(\Omega) \text { with compact imbeddings. }
$$

This fact implies the following important and useful imbeddings:

$$
\begin{gathered}
W^{\alpha}(0, T) \subset C\left([0, T] ; L^{2}(\Omega)\right) \quad \text { with continuous imbedding, and } \\
W^{\alpha}(0, T) \subset L^{\alpha}\left(0, T ; L^{2}(\Omega)\right) \quad \text { with compact imbedding. }
\end{gathered}
$$

Moreover, the following formula of integration by parts with respect to $t$ can be proved

$$
\int_{t_{0}}^{t_{1}}\left(y_{t}(s), \hat{y}(s)\right) d s+\int_{t_{0}}^{t_{1}}\left(y(s), \hat{y}_{t}(s)\right) d s=\int_{\Omega} y\left(x, t_{1}\right) \hat{y}\left(x, t_{1}\right) d x-\int_{\Omega} y\left(x, t_{0}\right) \hat{y}\left(x, t_{0}\right) d x
$$

for each $y, \hat{y} \in W^{\alpha}(0, T)$ and $t_{0}, t_{1} \in[0, T]$, where $(\cdot, \cdot)$ denotes the duality product between $W_{0}^{1, \alpha}(\Omega)$ and $W^{-1, \alpha^{\prime}}(\Omega)$; see [12].

Next, let us establish the existence and uniqueness of solution for the problem (2.1):

Theorem 2.1 Let (2.2)-(2.7) hold. Then, for each $y_{0} \in L^{\infty}(\Omega)$ and $u \in L^{r}\left(Q_{T}\right)$ with

$$
\begin{cases}r \geq \max \left\{2, \alpha^{\prime}\right\} & \text { if } n=1 \\ r>\frac{\alpha(n+2)}{\alpha(n+2)-2 n} & \text { if } n \geq 2 \text { and } \alpha \leq \frac{4 n}{n+2} \\ r \geq 2 & \text { if } n \geq 2 \text { and } \alpha>\frac{4 n}{n+2}\end{cases}
$$

there exists a unique solution $y_{u}$ of problem (2.1) in the space $W^{\alpha}(0, T) \cap L^{\infty}\left(Q_{T}\right)$. Moreover, there exists a constant $C_{1}$ depending on $\left\|y_{0}\right\|_{L^{\infty}(\Omega)}$ and $\|u\|_{L^{r}\left(Q_{T}\right)}$ such that

$$
\left\|y_{u}\right\|_{W^{\alpha}(0, T)}+\left\|y_{u}\right\|_{L^{\infty}\left(Q_{T}\right)} \leq C_{1}
$$

Remark 2.1 a) In any case, we have that $r \geq \alpha^{\prime}$ and then

$$
L^{r}\left(Q_{T}\right) \subset L^{\alpha^{\prime}}\left(Q_{T}\right) \subset L^{\alpha^{\prime}}\left(0, T ; W^{-1, \alpha^{\prime}}(\Omega)\right)
$$

b) By (2.9), the initial condition $y(x, 0)=y_{0}(x)$ makes sense. 
c) The requirement of $\alpha$ being strictly greater than $2 n /(n+2)$ is necessary in order to guarantee the boundedness of the solution.

Proof. Firstly, let us suppose that instead of (2.6) it holds

$$
\left(a_{0}(x, t, y)-a_{0}\left(x, t, y^{\prime}\right)\right)\left(y-y^{\prime}\right) \geq 0 \quad \text { and } \quad\left|a_{0}(x, t, y)\right| \leq \Lambda_{3}
$$

for some $\Lambda_{3}>0$ and all $(x, t) \in Q_{T}$ and $y, y^{\prime} \in \mathbb{R}$.

In this case, we are in the standard setting and the existence of a unique solution of (2.1) in $W^{\alpha}(0, T)$ is a consequence of [12, Theorem 1.2 bis, Chapter 2], Remark 2.1-a) and the hypothesis $\alpha>2 n /(n+2)$.

Furthermore, taking into account the assumption (2.12), that $\alpha>2 n /(n+2)$ and the monotonicity of $a_{0}$ with respect to $y$, we can apply the classical de Giorgi-Moser techniques (see [10]) to deduce the boundedness of the absolute value of the solution in $Q_{T}$ by a positive constant independent of $\Lambda_{3}$.

In the general case, for each $M>0$ we introduce the following truncation

$$
a_{0}^{M}(x, t, y)= \begin{cases}a_{0}(x, t, M) & \text { if } y \geq M \\ a_{0}(x, t, y) & \text { if } y \in[-M, M] \\ a_{0}(x, t,-M) & \text { if } y \leq-M\end{cases}
$$

which satisfies the conditions (2.14). Therefore, given $M>0$ we can consider the problem (2.1) with $a_{0}$ being replaced by $a_{0}^{M}$. Then, there exists a unique solution $y_{M} \in W^{\alpha}(0, T)$ of this problem and, moreover, there exists a positive constant $C_{0}$ independent of $M$ such that

$$
\left\|y_{M}\right\|_{L^{\infty}\left(Q_{T}\right)} \leq C_{0} \quad \forall M>0 .
$$

Hence, for any $M>C_{0}, y_{M}$ is independent of $M$ and it is a solution of (2.1). It is immediate to show that within the space $W^{\alpha}(0, T) \cap L^{\infty}\left(Q_{T}\right)$ the solution of (2.1) is unique. Finally, the estimate (2.13) can be obtained in a straightforward way.

Next, we prove the continuous dependence of the solution with respect to the data:

Corollary 2.1 Let us designate by $y_{m}$ the solution of (2.1) corresponding to $\left(y_{0}, u_{m}\right) \in L^{\infty}(\Omega) \times$ $L^{r}\left(Q_{T}\right)$ and suppose that $u_{m} \rightarrow u$ weakly in $L^{r}\left(Q_{T}\right)$ (weakly if $r=+\infty$ ) as $m \rightarrow+\infty$. Then,

$$
y_{m} \rightarrow y_{u} \quad \text { in } \quad L^{\alpha}\left(0, T ; W_{0}^{1, \alpha}(\Omega)\right) \cap C\left([0, T] ; L^{2}(\Omega)\right) \cap L^{q}\left(Q_{T}\right)
$$

for all $q \in[1,+\infty)$.

Proof. By virtue of the estimate (2.13), there exists a constant $C_{1}$ such that

$$
\left\|y_{m}\right\|_{W^{\alpha}(0, T)}+\left\|y_{m}\right\|_{L^{\infty}\left(Q_{T}\right)} \leq C_{1} \quad \forall m \in \mathbb{N} .
$$

Applying the relations satisfied by $y_{m}$ and $y_{u}$ to the element $y_{m}-y_{u}$, substrating them and integrating over $(0, t)$, for each $t \in(0, T]$ we have

$$
\begin{gathered}
\int_{0}^{t}\left(\frac{\partial y_{m}}{\partial t}(s)-\frac{\partial y_{u}}{\partial t}(s), y_{m}(s)-y_{u}(s)\right) d s+\int_{Q_{t}}\left(a\left(x, s, \nabla_{x} y_{m}\right)-a\left(x, s, \nabla_{x} y_{u}\right)\right)^{T}\left(\nabla_{x} y_{m}-\nabla_{x} y_{u}\right) d x d s+ \\
+\int_{Q_{t}}\left(a_{0}\left(x, s, y_{m}\right)-a_{0}\left(x, s, y_{u}\right)\right)\left(y_{m}-y_{u}\right) d x d s=\int_{Q_{t}}\left(u_{m}-u\right)\left(y_{m}-y_{u}\right) d x d s
\end{gathered}
$$


where $Q_{t}=\Omega \times(0, t)$.

If $\alpha \geq 2$, using now the formula of integration by parts (2.11) and taking into account the hypotheses (2.4) and (2.6), we obtain

$$
\begin{gathered}
\left\|y_{m}-y_{u}\right\|_{C\left([0, T] ; L^{2}(\Omega)\right)}^{2}+\left\|y_{m}-y_{u}\right\|_{L^{\alpha}\left(0, T ; W_{0}^{1, \alpha}(\Omega)\right)}^{\alpha} \leq \\
C_{2} \int_{Q_{T}}\left(u_{m}-u\right)\left(y_{m}-y_{u}\right) d x d s \leq C_{2}\left\|u_{m}-u\right\|_{W^{\alpha}(0, T)^{\prime}}\left\|y_{m}-y_{u}\right\|_{W^{\alpha}(0, T)} \rightarrow 0 \quad \text { as } m \rightarrow+\infty
\end{gathered}
$$

thanks to (2.15) and the imbeddings

$$
L^{r}\left(Q_{T}\right) \subset L^{\alpha^{\prime}}\left(0, T ; L^{2}(\Omega)\right) \subset W^{\alpha}(0, T)^{\prime},
$$

the second one being compact by duality of $(2.10)$, if $W^{\alpha}(0, T)^{\prime}$ denotes the dual space of $W^{\alpha}(0, T)$.

Because of the uniqueness of the limit, the whole sequence $\left\{y_{m}\right\}_{m \in \mathbf{N}}$ converges to $y_{u}$. Finally, the convergence in $L^{q}\left(Q_{T}\right)$ for all $q \in[1,+\infty)$ is again a consequence of (2.10) and (2.15).

For $\alpha<2$ the argumentation is similar, using that

$$
\begin{gathered}
\int_{Q_{T}}\left(a\left(x, s, \nabla_{x} y_{m}\right)-a\left(x, s, \nabla_{x} y_{u}\right)\right)^{T}\left(\nabla_{x} y_{m}-\nabla_{x} y_{u}\right) d x d s \geq \\
\mu_{1}\left\|1+\left|\nabla_{x} y_{m}\right|+\left|\nabla_{x} y_{u}\right|\right\|_{L^{\alpha}\left(Q_{T}\right)}^{\alpha-2}\left\|y_{m}-y_{u}\right\|_{L^{\alpha}\left(0, T ; W_{0}^{1, \alpha}(\Omega)\right)}^{2} \geq \\
\geq \frac{\mu_{1}}{\left(m\left(Q_{T}\right)^{1 / \alpha}+\left\|\nabla_{x} y_{u}\right\|_{L^{\alpha}\left(Q_{T}\right)}+C_{1}\right)^{2-\alpha}}\left\|y_{m}-y_{u}\right\|_{L^{\alpha}\left(0, T ; W_{0}^{1, \alpha}(\Omega)\right)}^{2}
\end{gathered}
$$

\section{The optimal control problem}

Let us consider a fixed element $y_{0} \in L^{\infty}(\Omega)$. We are concerned with the following control problem:

$$
(P) \min _{u \in \mathbf{K}} J(u)
$$

where $\mathbb{K}$ is a nonempty, convex and closed subset of $L^{r}\left(Q_{T}\right)$ (weak ${ }^{\star}$ closed, if $r=+\infty$ ), the cost functional is given by

$$
J(u)=\int_{Q_{T}} L\left(x, t, y_{u}(x, t), u(x, t)\right) d x d t,
$$

and $L: Q_{T} \times \mathbb{R} \times \mathbb{R} \longrightarrow \mathbb{R}$ is a measurable function satisfying

$$
L(x, t, \cdot, \cdot) \in C^{1}\left(\mathbb{R}^{2}\right) \quad \text { for each }(x, t) \in Q_{T}, \quad L(\cdot, \cdot, 0,0) \in L^{1}\left(Q_{T}\right) \text { and }
$$

moreover, for each $M>0$, there exist positive functions $h_{0}^{M} \in L^{r^{\prime}}\left(Q_{T}\right)$, where $r^{\prime}=\frac{r}{r-1}$, $h_{1}^{M} \in L^{2}\left(Q_{T}\right)$, and a constant $C_{M}>0$ such that

$$
\begin{aligned}
\left|\frac{\partial L}{\partial u}(x, t, y, u)\right| & \leq h_{0}^{M}(x, t)+C_{M}|u|^{r-1} \\
\left|\frac{\partial L}{\partial y}(x, t, y, u)\right| & \leq h_{1}^{M}(x, t)+C_{M}|u|^{r / 2}
\end{aligned}
$$

for all $(x, t, y, u) \in Q_{T} \times[-M, M] \times \mathbb{R}$. For $r=+\infty$, we must replace $|u|^{r-1}$ and $|u|^{r / 2}$ by $\varphi(|u|)$, where $\varphi$ is a positive increasing function.

With the aid of Theorem 2.1 and Corollary 2.1, the existence of solutions of $(P)$ can be established in a standard way: 
Theorem 3.1 Let (2.2)-(2.7), (2.12) and (3.1)-(3.3) hold together with the following additional conditions:

a) the function $L(x, t, y, \cdot)$ is convex for each $(x, t, y) \in Q_{T} \times \mathbb{R}$ and

b) there exists $u_{0} \in \mathbb{K}$, such that the set $\left\{u \in \mathbb{K}: J(u) \leq J\left(u_{0}\right)\right\}$ is bounded in $L^{r}\left(Q_{T}\right)$.

Then, there exists at least one optimal control for problem $(P)$.

Proof. Thanks to the hypotheses and Theorem 2.1, it is easy to verify that $J(u)$ is finite for each $u \in L^{r}\left(Q_{T}\right)$.

Let $\left\{u_{m}\right\}_{m \in \mathbf{N}} \subset \mathbb{K}$ be a minimizing sequence and $\left\{y_{m}\right\}_{m \in \mathbf{N}}$ be the corresponding states, i.e. the solutions of $(2.1)$ corresponding to $\left(y_{0}, u_{m}\right)$. By condition $\left.\mathrm{b}\right)$, there exists a constant $C_{1}>0$, such that

$$
\left\|u_{m}\right\|_{L^{r}\left(Q_{T}\right)} \leq C_{1} \quad \forall m \in \mathbb{N}
$$

Hence, we may assume that

$$
\left.u_{m} \longrightarrow \bar{u} \text { weakly in } L^{r}\left(Q_{T}\right) \text { (weakly* if } r=+\infty\right)
$$

for some $\bar{u} \in \mathbb{K}$ (let us recall that $\mathbb{K}$ is weak closed in $L^{r}\left(Q_{T}\right)$, resp. weak ${ }^{\star}$ closed, if $r=+\infty$.)

Combining the conclusions of Theorem 2.1 and Corollary 2.1, we can deduce that

$$
\begin{gathered}
\left\|y_{m}\right\|_{L^{\infty}\left(Q_{T}\right)} \leq M \quad \forall m \in \mathbb{N} \quad \text { and } \\
y_{m} \longrightarrow y_{\bar{u}} \quad \text { in } L^{2}\left(Q_{T}\right) \quad \text { as } m \rightarrow+\infty
\end{gathered}
$$

for some positive constant $M$.

On the other hand, by Mazur's theorem (see for instance [8]), we can find a sequence of convex combinations $\left\{v_{m}\right\}_{m \in \mathbf{N}}$, i.e.

$$
v_{m}=\sum_{l=m}^{N(m)} \lambda_{m}^{l} u_{l} \quad \text { with } \quad \sum_{l=m}^{N(m)} \lambda_{m}^{l}=1 \quad \text { and } \quad \lambda_{m}^{l} \geq 0, \quad m \leq l \leq N(m),
$$

such that

$$
v_{m} \longrightarrow \bar{u} \quad \text { in } L^{r}\left(Q_{T}\right) \quad \text { as } m \rightarrow+\infty .
$$

In the case $r=+\infty$, this convergence remains true in $L^{q}\left(Q_{T}\right)$ for any $q<+\infty$.

Using now the convexity of $L$ with respect to $u$, the Dominated Convergence Theorem and (3.2)-(3.3), we obtain

$$
\begin{gathered}
J(\bar{u})=\int_{Q_{T}} L\left(x, t, y_{\bar{u}}, \bar{u}\right) d x d t=\lim _{m} \int_{Q_{T}} L\left(x, t, y_{\bar{u}}, v_{m}\right) d x d t \leq \\
\leq \lim _{m} \sum_{l=m}^{N(m)} \lambda_{m}^{l} \int_{Q_{T}} L\left(x, t, y_{\bar{u}}, u_{l}\right) d x d t \leq \\
\leq \lim _{m} \sum_{l=m}^{N(m)} \lambda_{m}^{l}\left(J\left(u_{l}\right)+\int_{Q_{T}}\left|L\left(x, t, y_{l}, u_{l}\right)-L\left(x, t, y_{\bar{u}}, u_{l}\right)\right| d x d t\right) \leq \\
\leq \inf _{u \in \mathbf{K}} J(u)+\lim _{m} \sum_{l=m}^{N(m)} \lambda_{m}^{l} \int_{Q_{T}}\left(h_{1}^{M}(x, t)+C_{M}\left|u_{l}\right|^{r / 2}\right)\left|y_{l}-y_{\bar{u}}\right| d x d t \leq
\end{gathered}
$$




$$
\begin{gathered}
\leq \inf _{u \in \mathbf{K}} J(u)+\lim _{m} \sum_{l=m}^{N(m)} \lambda_{m}^{l}\left(\left\|h_{1}^{M}\right\|_{L^{2}\left(Q_{T}\right)}+C_{M}\left\|u_{l}\right\|_{L^{2}\left(Q_{T}\right)}^{r / 2}\right)\left\|y_{l}-y_{\bar{u}}\right\|_{L^{2}\left(Q_{T}\right)} \\
=\inf _{u \in \mathbf{K}} J(u) .
\end{gathered}
$$

That means $\bar{u}$ is an optimal control for $(P)$.

Remark 3.1 a) In the existence theory of optimal controls, convexity conditions are usually assumed. Condition a) is one such condition. We should point out here that if one only discusses the existence of optimal controls the differentiability conditions on $L$ can be removed. Consequently, (3.2)-(3.3) can be replaced by some weaker conditions. Here, we do not intend to get into this generality since our main objective is to derive the optimality conditions, for which we need the differentiability of the function $L$ with respect to $y$ and $u$.

b) When $\mathbb{K}$ is bounded in $L^{r}\left(Q_{T}\right)$, condition b) is automatically true. If $\mathbb{K}$ is unbounded in $L^{r}\left(Q_{T}\right)$ with $r<+\infty$ to guarantee b) we may assume that for each $M>0$ there exist a constant $\hat{C}_{M}>0$ and a function $h_{2}^{M} \in L^{1}\left(Q_{T}\right)$, such that

$$
L(x, t, y, u) \geq \hat{C}_{M}|u|^{r}-h_{2}^{M}(x, t), \quad \forall(x, t, y, u) \in Q_{T} \times[-M, M] \times \mathbb{R}
$$

For $r=+\infty$, we have to assume $\mathbb{K}$ to be bounded.

\section{Sensitivity analysis}

As mentioned in $\S 1$, in order to derive some optimality conditions satisfied by an optimal control $\bar{u}$, we investigate the differentiability of the relation between the control and the state: $u \rightarrow y_{u}$. In this study some difficulties arise by the (possible) non-uniqueness of solution for the linearized problem that is (in general) of degenerate type. Nevertheless, there are some special situations in which this difficulty does not appear. These can be summarized as follows:

Theorem 4.1 Let us assume (2.2)-(2.7), (2.12) and consider $F: L^{r}\left(Q_{T}\right) \longrightarrow L^{2}\left(0, T ; H_{0}^{1}(\Omega)\right)$ (resp. $L^{\alpha}\left(0, T ; W_{0}^{1, \alpha}(\Omega)\right)$, if $\alpha<2$ ) the functional defined by $F(u)=y_{u}$.

a) If $\alpha=2$, then $F$ is Gâteaux differentiable.

b) If $\alpha \neq 2$ and $k \neq 0$, then $F$ is Gâteaux differentiable at the points $u \in L^{r}\left(Q_{T}\right)$ such that $\left|\nabla_{x} y_{u}\right| \in L^{\infty}\left(Q_{T}\right)$.

Moreover if $z=D F(u) v$, then $z \in W^{2}(0, T)$ and it is the unique solution in this space of the problem

$$
\begin{cases}z_{t}-\operatorname{div}_{x}\left\{\frac{\partial a}{\partial \eta}\left(x, t, \nabla_{x} y_{u}\right) \nabla_{x} z\right\}+\frac{\partial a_{0}}{\partial y}\left(x, t, y_{u}\right) z=v & \text { in } Q_{T} \\ z(x, t)=0 & \text { on } \Sigma, \\ z(x, 0)=0 & \text { in } \Omega .\end{cases}
$$

Remark 4.1 Under the hypotheses of the theorem it is immediate to verify the boundedness of the coefficients of the linearized operator and deduce the existence and uniqueness of solution for (4.1) in $W^{2}(0, T)$. Let us remember that in any case $r \geq 2$ and hence $L^{r}\left(Q_{T}\right) \subset L^{2}\left(Q_{T}\right)$. 
Proof. Given $u, v \in L^{r}\left(Q_{T}\right)$ and $0<\lambda<1$, we consider the problem

$$
\begin{cases}y_{t}(x, t)+A y(x, t)=u(x, t)+\lambda v(x, t) & \text { in } Q_{T} \\ y(x, t)=0 & \text { on } \Sigma \\ y(x, 0)=y_{0}(x) & \text { in } \Omega\end{cases}
$$

By Theorem 2.1, for each $\lambda$ there exists a unique solution $y^{\lambda} \in W^{\alpha}(0, T) \cap L^{\infty}\left(Q_{T}\right)$. Moreover,

$$
\left\|y^{\lambda}\right\|_{L^{\infty}\left(Q_{T}\right)} \leq C_{1} \quad \forall \lambda \in(0,1)
$$

for some positive constant $C_{1}>0$ and thanks to Corollary 2.1

$$
y^{\lambda} \longrightarrow y_{u} \quad \text { in } L^{\alpha}\left(0, T ; W_{0}^{1, \alpha}(\Omega)\right) \quad \text { as } \lambda \rightarrow 0
$$

Now, let us introduce the function

$$
z^{\lambda}=\frac{y^{\lambda}-y_{u}}{\lambda}
$$

To complete the proof, we must deduce the converge of $\left\{z^{\lambda}\right\}$ towards $z$ in $L^{2}\left(0, T ; H_{0}^{1}(\Omega)\right)$ (resp. $L^{\alpha}\left(0, T ; W_{0}^{1, \alpha}(\Omega)\right)$, if $\left.\alpha<2\right)$, where $z$ designates the unique solution of $(4.1)$ in $W^{2}(0, T)$.

Let us begin by considering the case $\alpha \geq 2$ (with $k \neq 0$ and $\left|\nabla_{x} y_{u}\right| \in L^{\infty}\left(Q_{T}\right)$ when $\alpha>2$ ). At the end, we will point out the differences in the case $\alpha<2$.

It will be convenient to divide the argumentation into several steps.

Step 1. The sequence $\left\{z^{\lambda}\right\}_{\lambda>0}$ is bounded in $L^{2}\left(0, T ; H_{0}^{1}(\Omega)\right)$.

Proof of Step 1. Taking into account (4.2), it is clear that for each $\lambda \in(0,1)$

$$
\begin{cases}z_{t}^{\lambda}-\operatorname{div}_{x}\left\{\frac{a\left(x, t, \nabla_{x} y^{\lambda}\right)-a\left(x, t, \nabla_{x} y_{u}\right)}{\lambda}\right\}+\frac{a_{0}\left(x, t, y^{\lambda}\right)-a_{0}\left(x, t, y_{u}\right)}{\lambda}=v & \text { in } Q_{T}, \\ z^{\lambda}(x, t)=0 & \text { on } \Sigma, \\ z^{\lambda}(x, 0)=0 & \text { in } \Omega .\end{cases}
$$

Applying (4.5) to $z^{\lambda}$ and integrating by parts we obtain

$$
\begin{gathered}
\frac{1}{2}\left\|z^{\lambda}(\cdot, T)\right\|_{L^{2}(\Omega)}^{2}+\int_{Q_{T}} \frac{1}{\lambda}\left(a\left(x, t, \nabla_{x} y^{\lambda}\right)-a\left(x, t, \nabla_{x} y_{u}\right)\right)^{T} \nabla_{x} z^{\lambda} d x d t+ \\
+\int_{Q_{T}} \frac{1}{\lambda}\left(a_{0}\left(x, t, y^{\lambda}\right)-a_{0}\left(x, t, y_{u}\right)\right) z^{\lambda} d x d t=\int_{Q_{T}} v z^{\lambda} d x d t
\end{gathered}
$$

By using now the Mean Value Theorem, we get

$$
\begin{gathered}
\frac{1}{2}\left\|z^{\lambda}(\cdot, T)\right\|_{L^{2}(\Omega)}^{2}+\int_{Q_{T}}\left(\nabla_{x} z^{\lambda}\right)^{T} \frac{\partial a}{\partial \eta}\left(x, t, w^{\lambda}\right) \nabla_{x} z^{\lambda} d x d t+ \\
+\int_{Q_{T}} \frac{\partial a_{0}}{\partial y}\left(x, t, w_{0}^{\lambda}\right)\left|z^{\lambda}\right|^{2} d x d t=\int_{Q_{T}} v z^{\lambda} d x d t
\end{gathered}
$$

where $w^{\lambda}=\nabla_{x} y_{u}+\theta \nabla_{x}\left(y^{\lambda}-y_{u}\right)$ and $w_{0}^{\lambda}=y_{u}+\theta_{0}\left(y^{\lambda}-y_{u}\right)$, for some $\theta$ and $\theta_{0}$ depending on $x, t, \lambda$ and belonging to $(0,1)$. 
Together with the hypotheses (2.4) and (2.6), this implies

$$
\begin{gathered}
\Lambda_{1} k^{\alpha-2}\left\|z^{\lambda}\right\|_{L^{2}\left(0, T ; H_{0}^{1}(\Omega)\right)}^{2} \leq \Lambda_{1} \int_{Q_{T}}\left(k+\left|w^{\lambda}\right|\right)^{\alpha-2}\left|\nabla_{x} z^{\lambda}\right|^{2} d x d t \leq \\
\|v\|_{L^{2}\left(Q_{T}\right)}\left\|z^{\lambda}\right\|_{L^{2}\left(Q_{T}\right)} \leq C_{2}\|v\|_{L^{2}\left(Q_{T}\right)}\left\|z^{\lambda}\right\|_{L^{2}\left(0, T ; H_{0}^{1}(\Omega)\right)},
\end{gathered}
$$

from which it follows the boundedness of the sequence $\left\{z^{\lambda}\right\}$ in $L^{2}\left(0, T ; H_{0}^{1}(\Omega)\right)$.

Step 2. The sequence $\left\{z^{\lambda}\right\}_{\lambda>0}$ converges weakly in $L^{2}\left(0, T ; H_{0}^{1}(\Omega)\right)$ to the unique solution of Problem (4.1) in $W^{2}(0, T)$.

Proof of Step 2. By Step 1 we know that there exist a subsequence of $\left\{z^{\lambda}\right\}_{\lambda>0}$, that we denote in the same way, and an element $z \in L^{2}\left(0, T ; H_{0}^{1}(\Omega)\right)$ such that

$$
z^{\lambda} \longrightarrow z \quad \text { weakly in } L^{2}\left(0, T ; H_{0}^{1}(\Omega)\right) \quad \text { as } \lambda \rightarrow 0 .
$$

We will show that $z$ is the unique solution in $W^{2}(0, T)$ of Problem (4.1) (which implies the conclusion of Step 2) by proving that

$$
\begin{gathered}
\frac{a\left(x, t, \nabla_{x} y^{\lambda}\right)-a\left(x, t, \nabla_{x} y_{u}\right)}{\lambda} \longrightarrow \frac{\partial a}{\partial \eta}\left(x, t, \nabla_{x} y_{u}\right) \nabla_{x} z \quad \text { weakly in } L^{\alpha^{\prime}}\left(Q_{T}\right)^{n}, \\
\frac{a_{0}\left(x, t, y^{\lambda}\right)-a_{0}\left(x, t, y_{u}\right)}{\lambda} \longrightarrow \frac{\partial a_{0}}{\partial y}\left(x, t, y_{u}\right) z \quad \text { weakly in } L^{2}\left(Q_{T}\right)
\end{gathered}
$$

as $\lambda \rightarrow 0$ and passing to the limit in (4.5).

Previously, let us establish a crucial estimation. Thanks to (2.4) we have that

$$
\int_{Q_{T}}\left(a\left(x, t, \nabla_{x} y^{\lambda}\right)-a\left(x, t, \nabla_{x} y_{u}\right)\right)^{T}\left(\nabla_{x} y^{\lambda}-\nabla_{x} y_{u}\right) d x d t \geq \mu_{1}\left\|y^{\lambda}-y_{u}\right\|_{L^{\alpha}\left(0, T ; W_{0}^{1, \alpha}(\Omega)\right)}^{\alpha}
$$

Therefore, by virtue of (4.5), (4.7) and (4.11) we get

$$
\frac{1}{\lambda^{2}}\left\|y^{\lambda}-y_{u}\right\|_{L^{\alpha}\left(0, T ; W_{0}^{1, \alpha}(\Omega)\right)}^{\alpha} \leq C_{3}\|v\|_{L^{2}\left(Q_{T}\right)}^{2}
$$

Given $1 \leq j \leq n$, using again the Mean Value Theorem, we get

$$
\frac{a_{j}\left(x, t, \nabla_{x} y^{\lambda}\right)-a_{j}\left(x, t, \nabla_{x} y_{u}\right)}{\lambda}=\sum_{i=1}^{n} \frac{\partial a_{j}}{\partial \eta_{i}}\left(x, t, \hat{w}_{j}^{\lambda}\right) \frac{\partial z^{\lambda}}{\partial x_{i}}
$$

where $\hat{w}_{j}^{\lambda}=\nabla_{x} y_{u}+\hat{\theta}_{j} \nabla_{x}\left(y^{\lambda}-y_{u}\right)$, for some $\hat{\theta}_{j}$ depending on $x, t, \lambda$ and belonging to $(0,1)$.

Combining (4.12) and the conclusion of previous step with the hypothesis (2.5), we derive that

$$
\begin{gathered}
\int_{Q_{T}}\left|\frac{\partial a_{j}}{\partial \eta_{i}}\left(x, t, \hat{w}_{j}^{\lambda}\right)\right|\left|\frac{\partial z^{\lambda}}{\partial x_{i}}\right|^{2} d x d t \leq \Lambda_{2} \int_{Q_{T}}\left(k+\left|\hat{w}_{j}^{\lambda}\right|\right)^{\alpha-2}\left|\frac{\partial z^{\lambda}}{\partial x_{i}}\right|^{2} d x d t \leq \\
\leq \Lambda_{2} 2^{\alpha-2}\left(\| k+\left.\left|\nabla_{x} y_{u}\right|\right|_{L^{\infty}\left(Q_{T}\right)} ^{\alpha-2} \int_{Q_{T}}\left|\frac{\partial z^{\lambda}}{\partial x_{i}}\right|^{2} d x d t+\frac{1}{\lambda^{2}} \int_{Q_{T}}\left|\nabla_{x}\left(y^{\lambda}-y_{u}\right)\right|^{\alpha} d x d t\right) \leq C_{4}
\end{gathered}
$$

In particular this implies that

$$
\left\{\left(\frac{\partial a_{j}}{\partial \eta_{i}}\left(x, t, \hat{w}_{j}^{\lambda}\right)\right)^{m /(2 m+1)} \frac{\partial z^{\lambda}}{\partial x_{i}}\right\} \text { is bounded in } L^{2}\left(Q_{T}\right)
$$


for each $m \in \mathbb{N}$ and for all $i, j \in\{1, \ldots, n\}$ (let us notice that $\frac{\partial a_{j}}{\partial \eta_{i}}$ can take both positive or negative values when $i \neq j$ ).

Moreover, a simple application of the Dominated Convergence Theorem (taking a new subsequence, if necessary) yields

$$
\left(\frac{\partial a_{j}}{\partial \eta_{i}}\left(x, t, \hat{w}_{j}^{\lambda}\right)\right)^{m /(2 m+1)} \longrightarrow\left(\frac{\partial a_{j}}{\partial \eta_{i}}\left(x, t, \nabla_{x} y_{u}\right)\right)^{m /(2 m+1)} \quad \text { in } L^{2}\left(Q_{T}\right)
$$

and for $m \geq \frac{\alpha-4}{4}$

$$
\left(\frac{\partial a_{j}}{\partial \eta_{i}}\left(x, t, \hat{w}_{j}^{\lambda}\right)\right)^{(m+1) /(2 m+1)} \longrightarrow\left(\frac{\partial a_{j}}{\partial \eta_{i}}\left(x, t, \nabla_{x} y_{u}\right)\right)^{(m+1) /(2 m+1)} \quad \text { in } L^{2}\left(Q_{T}\right) .
$$

From (4.15) and (4.16), it follows that

$$
\left(\frac{\partial a_{j}}{\partial \eta_{i}}\left(x, t, \hat{w}_{j}^{\lambda}\right)\right)^{m /(2 m+1)} \frac{\partial z^{\lambda}}{\partial x_{i}} \longrightarrow\left(\frac{\partial a_{j}}{\partial \eta_{i}}\left(x, t, \nabla_{x} y_{u}\right)\right)^{m /(2 m+1)} \frac{\partial z}{\partial x_{i}} \quad \text { weakly in } L^{2}\left(Q_{T}\right)
$$

as $\lambda \rightarrow 0$.

Using now (2.5), (4.4), (4.14) and Young's inequality we can deduce

$$
\begin{gathered}
\int_{Q_{T}}\left|\left(\frac{\partial a_{j}}{\partial \eta_{i}}\left(x, t, \hat{w}_{j}^{\lambda}\right)\right) \frac{\partial z^{\lambda}}{\partial x_{i}}\right|^{\alpha^{\prime}} d x d t \leq \Lambda_{2}^{\alpha^{\prime}} \int_{Q_{T}}\left(k+\left|\hat{w}_{j}^{\lambda}\right|\right)^{(\alpha-2) \alpha^{\prime}}\left|\frac{\partial z^{\lambda}}{\partial x_{i}}\right|^{\alpha^{\prime}} d x d t= \\
\Lambda_{2}^{\alpha^{\prime}} \int_{Q_{T}}\left(k+\left|\hat{w}_{j}^{\lambda}\right|\right)^{(\alpha-2) \frac{\alpha^{\prime}}{2}}\left(k+\left|\hat{w}_{j}^{\lambda}\right|\right)^{(\alpha-2) \frac{\alpha^{\prime}}{2}}\left|\frac{\partial z^{\lambda}}{\partial x_{i}}\right|^{\alpha^{\prime}} d x d t \leq \\
\leq C_{5}\left(\int_{Q_{T}}\left(k+\left|\nabla_{x} y_{u}\right|+\left|\nabla_{x} y^{\lambda}\right|\right)^{\alpha} d x d t+\int_{Q_{T}}\left(k+\left|\hat{w}_{j}^{\lambda}\right|\right)^{\alpha-2}\left|\frac{\partial z^{\lambda}}{\partial x_{i}}\right|^{2} d x d t\right) \leq C_{6}
\end{gathered}
$$

for all $\lambda \in(0,1)$ and $i, j \in\{1, \ldots, n\}$. Applying the following decomposition

$$
\left(\frac{\partial a_{j}}{\partial \eta_{i}}\left(x, t, \hat{w}_{j}^{\lambda}\right)\right) \frac{\partial z^{\lambda}}{\partial x_{i}}=\left(\frac{\partial a_{j}}{\partial \eta_{i}}\left(x, t, \hat{w}_{j}^{\lambda}\right)\right)^{(m+1) /(2 m+1)} \cdot\left(\frac{\partial a_{j}}{\partial \eta_{i}}\left(x, t, \hat{w}_{j}^{\lambda}\right)\right)^{m /(2 m+1)} \frac{\partial z^{\lambda}}{\partial x_{i}}
$$

together with (4.17)-(4.19) we conclude that

$$
\frac{\partial a_{j}}{\partial \eta_{i}}\left(x, t, \hat{w}_{j}^{\lambda}\right) \frac{\partial z^{\lambda}}{\partial x_{i}} \longrightarrow \frac{\partial a_{j}}{\partial \eta_{i}}\left(x, t, \nabla_{x} y_{u}\right) \frac{\partial z}{\partial x_{i}} \quad \text { weakly in } L^{\alpha^{\prime}}\left(Q_{T}\right) \text { as } \lambda \rightarrow 0
$$

for all $i, j \in\{1, \ldots, n\}$, and consequently (4.9).

Of course, for $\alpha=2$ the estimate (4.19) and the convergence (4.20) are obtained directly from (2.2), (2.5), (4.4) and (4.8).

Similarly, the hypotheses (2.3) and (2.6) together with the relations (4.4) and (4.6) allow us to obtain

$$
\frac{a_{0}\left(x, t, y^{\lambda}\right)-a_{0}\left(x, t, y_{u}\right)}{\lambda}=\frac{\partial a_{0}}{\partial y}\left(x, t, w_{0}^{\lambda}\right) z^{\lambda} \longrightarrow \frac{\partial a_{0}}{\partial y}\left(x, t, y_{u}\right) z \quad \text { weakly in } L^{2}\left(Q_{T}\right) \quad \text { as } \lambda \rightarrow 0
$$


With the aid of (4.8)-(4.10), we can pass to the limit in (4.5) as $\lambda \rightarrow 0$ and get that

$$
z_{t}-\operatorname{div}_{x}\left\{\frac{\partial a}{\partial \eta}\left(x, t, \nabla_{x} y_{u}\right) \nabla_{x} z\right\}+\frac{\partial a_{0}}{\partial y}\left(x, t, y_{u}\right) z=v \quad \text { in } \quad Q_{T}
$$

From this relation and the assumption (2.5) with $\alpha=2$ or $\left|\nabla_{x} y_{u}\right| \in L^{\infty}\left(Q_{T}\right)$ if $\alpha>2$ and $k \neq 0$, we deduce that

$$
\frac{\partial a_{j}}{\partial \eta_{i}}\left(x, t, \nabla_{x} y_{u}\right) \in L^{\infty}\left(Q_{T}\right) \quad \forall i, j \in\{1, \ldots, n\}
$$

Consequently, $z_{t} \in L^{2}\left(0, T ; H^{-1}(\Omega)\right)$ and hence, $z \in W^{2}(0, T) \subset C\left([0, T] ; L^{2}(\Omega)\right)$.

Finally, by virtue of (4.8)-(4.10) we also have that

$$
z^{\lambda} \longrightarrow z \quad \text { weakly in } W^{1, \alpha^{\prime}}\left(0, T ; W^{-1, \alpha^{\prime}}(\Omega)\right)
$$

Using now the continuity of imbedding (2.8) we derive that

$$
z^{\lambda} \longrightarrow z \quad \text { weakly in } C\left([0, T] ; W^{-1, \alpha^{\prime}}(\Omega)\right)
$$

and, in particular

$$
0=z^{\lambda}(\cdot, 0) \longrightarrow z(\cdot, 0) \quad \text { weakly in } W^{-1, \alpha^{\prime}}(\Omega)
$$

This implies that $z(\cdot, 0)=0$ and therefore, $z$ is the unique solution in $W^{2}(0, T)$ of problem (4.1).

Step 3. The sequence $\left\{z^{\lambda}\right\}_{\lambda>0}$ converges strongly in $L^{2}\left(0, T ; H_{0}^{1}(\Omega)\right)$ to the unique solution of Problem (4.1) in $W^{2}(0, T)$.

Using the estimate (4.6) and arguing as above, we deduce

$$
z^{\lambda}(\cdot, T) \longrightarrow z(\cdot, T) \text { weakly in } L^{2}(\Omega) .
$$

Now, from (4.6), (4.22) and (4.23), it follows

$$
\lim _{\lambda \rightarrow 0} \int_{Q_{T}}\left(\nabla_{x} z^{\lambda}\right)^{T} \frac{\partial a}{\partial \eta}\left(x, t, w^{\lambda}\right) \nabla_{x} z^{\lambda} d x d t=\int_{Q_{T}}\left(\nabla_{x} z\right)^{T} \frac{\partial a}{\partial \eta}\left(x, t, \nabla_{x} y_{u}\right) \nabla_{x} z d x d t
$$

The last convergence implies $\nabla_{x} z^{\lambda} \rightarrow \nabla_{x} z$ in $L^{2}\left(Q_{T}\right)^{n}$, as it can be proved by the same argumentation as in [4, Theorem 3.1, Step 3].

Finally, from (4.7), we get

$$
\|z\|_{L^{2}\left(0, T ; H_{0}^{1}(\Omega)\right)} \leq \frac{C_{2}}{\Lambda_{1} k^{\alpha-2}}\|v\|_{L^{2}\left(Q_{T}\right)} \leq C_{7}\|v\|_{L^{r}\left(Q_{T}\right)}
$$

which implies the continuity of $D F(u): L^{r}\left(Q_{T}\right) \longrightarrow L^{2}\left(0, T ; H_{0}^{1}(\Omega)\right)$, and therefore the Gâteaux differentiability of $F$ when $\alpha=2$ or $\alpha>2, k \neq 0$ and $\left|\nabla_{x} y_{u}\right| \in L^{\infty}\left(Q_{T}\right)$.

In the case $\alpha<2, k \neq 0$ and $\left|\nabla_{x} y_{u}\right| \in L^{\infty}\left(Q_{T}\right)$ there are some minor changes in the argumentation that can be summarized as follows:

- The boundedness of the sequence $\left\{z^{\lambda}\right\}$ in $L^{\alpha}\left(0, T ; W_{0}^{1, \alpha}(\Omega)\right)$ can be obtained using (2.4), (4.4), (4.6) and the Hölder's inequality in the form

$$
\begin{gathered}
\Lambda_{1}\left\|z^{\lambda}\right\|_{L^{\alpha}\left(0, T ; W_{0}^{1, \alpha}(\Omega)\right)}^{2}\left\|\left(k+\left|w^{\lambda}\right|\right)\right\|_{L^{\alpha}\left(Q_{T}\right)}^{\alpha-2} \leq \Lambda_{1} \int_{Q_{T}} \frac{\left|\nabla_{x} z^{\lambda}\right|^{2}}{\left(k+\left|w^{\lambda}\right|\right)^{2-\alpha}} d x d t \leq \int_{Q_{T}} v z^{\lambda} d x d t \leq \\
\leq C_{8}\|v\|_{L^{\alpha^{\prime}\left(Q_{T}\right)}}\left\|z^{\lambda}\right\|_{L^{\alpha}\left(0, T ; W_{0}^{1, \alpha}(\Omega)\right)}
\end{gathered}
$$


- Given $z \in L^{\alpha}\left(0, T ; W_{0}^{1, \alpha}(\Omega)\right)$ such that

$$
z^{\lambda} \longrightarrow z \quad \text { weakly in } L^{\alpha}\left(0, T ; W_{0}^{1, \alpha}(\Omega)\right)
$$

(at least for a subsequence) from (4.4) and (4.24) it is immediate to deduce that

$$
\frac{\nabla_{x} z^{\lambda}}{\left(k+\left|w^{\lambda}\right|\right)^{\frac{2-\alpha}{2}}} \longrightarrow \frac{\nabla_{x} z}{\left(k+\left|\nabla_{x} y_{u}\right|\right)^{\frac{2-\alpha}{2}}} \quad \text { weakly in } L^{2}\left(Q_{T}\right)^{n} .
$$

Hence, since $\left|\nabla_{x} y_{u}\right| \in L^{\infty}\left(Q_{T}\right)$ we deduce that $z \in L^{2}\left(0, T ; H_{0}^{1}(\Omega)\right)$.

- Since $\alpha<2$, it is easily verified the weak convergences (4.20) and (4.21) in $L^{\alpha}\left(Q_{T}\right)$. We conclude as before that $z \in W^{2}(0, T)$ and it is the unique solution of problem (4.1) in this space.

\section{Optimality conditions}

In the sequel, we will assume that $\bar{u} \in \mathbb{K}$ is an optimal control for $(P)$ and $\bar{y}$ its associated state. With the aid of Theorem 4.1 we can easily obtain the first order optimality system when $\alpha=2$, which includes the semilinear case.

Theorem 5.1 Let us assume the conditions (2.2)-(2.7) (with $k \neq 0$ if $\alpha \neq 2$ ), (2.12) and (3.1)-(3.3). Let $\bar{u}$ be an optimal control for $(P)$ and $\bar{y} \in W^{\alpha}(0, T) \cap L^{\infty}\left(Q_{T}\right)$ the optimal state, i.e.

$$
\begin{cases}\bar{y}_{t}(x, t)+A \bar{y}(x, t)=\bar{u}(x, t) & \text { in } Q_{T}, \\ \bar{y}(x, t)=0 & \text { on } \Sigma, \\ \bar{y}(x, 0)=y_{0}(x) & \text { in } \Omega .\end{cases}
$$

Moreover, let us suppose that $\left|\nabla_{x} \bar{y}\right| \in L^{\infty}\left(Q_{T}\right)$ when $\alpha \neq 2$.

Then, there exists a unique element $\bar{p} \in W^{2}(0, T)$ such that

$$
\begin{cases}-\bar{p}_{t}-\operatorname{div}_{x}\left(\left[\frac{\partial a}{\partial \eta}\left(x, t, \nabla_{x} \bar{y}\right)\right]^{T} \nabla_{x} \bar{p}\right)+\frac{\partial a_{0}}{\partial y}(x, t, \bar{y}) \bar{p}=\frac{\partial L}{\partial y}(x, t, \bar{y}, \bar{u}) & \text { in } Q_{T} \\ \bar{p}(x, t)=0 & \text { on } \Sigma \\ \bar{p}(x, T)=0 & \text { in } \Omega\end{cases}
$$

and

$$
\int_{Q_{T}}\left(\bar{p}+\frac{\partial L}{\partial u}(x, t, \bar{y}, \bar{u})\right)(u-\bar{u}) d x d t \geq 0 \quad \forall u \in \mathbb{K}
$$

Proof. By virtue of hypotheses (3.1)-(3.3) together with the conclusion of Theorem 4.1, it is easy to prove that the cost functional $J$ is Gâteaux differentiable at $\bar{u}$ and

$$
J^{\prime}(\bar{u})(u-\bar{u})=\int_{Q_{T}} \frac{\partial L}{\partial y}(x, t, \bar{y}, \bar{u}) z d x d t+\int_{Q_{T}} \frac{\partial L}{\partial u}(x, t, \bar{y}, \bar{u})(u-\bar{u}) d x d t
$$

where $z=D F(\bar{u})(u-\bar{u}) \in W^{2}(0, T)$ with the notation of Theorem 4.1.

Furthermore, since $\mathbb{K}$ is a convex set it is well known that

$$
J^{\prime}(\bar{u})(u-\bar{u}) \geq 0 \quad \forall u \in \mathbb{K}
$$


Now let $\bar{p}$ be the unique solution of $(5.1)$ in $W^{2}(0, T)$ (notice that $\frac{\partial L}{\partial y}(\cdot, \cdot, \bar{y}, \bar{u}) \in L^{2}\left(Q_{T}\right)$ thanks to (3.3)). Then, using the formula of integration by parts (2.11) we have

$$
\begin{gathered}
\int_{Q_{T}} \frac{\partial L}{\partial y}(x, s, \bar{y}, \bar{u}) z d x d s=-\int_{0}^{T}\left(\bar{p}_{t}(s), z(s)\right) d s+ \\
+\int_{Q_{T}}\left(\nabla_{x} z\right)^{T}\left(\frac{\partial a}{\partial \eta}\left(x, s, \nabla_{x} \bar{y}\right)\right)^{T} \nabla_{x} \bar{p} d x d s+\int_{Q_{T}} \frac{\partial a_{0}}{\partial y}(x, s, \bar{y}) z \bar{p} d x d s= \\
=\int_{0}^{T}\left(z_{t}(s), \bar{p}(s)\right) d s+\int_{Q_{T}}\left(\nabla_{x} \bar{p}\right)^{T}\left(\frac{\partial a}{\partial \eta}\left(x, s, \nabla_{x} \bar{y}\right)\right) \nabla_{x} z d x d s+ \\
+\int_{Q_{T}} \frac{\partial a_{0}}{\partial y}(x, s, \bar{y}) z \bar{p} d x d s=\int_{Q_{T}} \bar{p}(u-\bar{u}) d x d s .
\end{gathered}
$$

Last equality follows from Theorem 4.1. Finally, combining (5.3)-(5.5) we obtain (5.2)

When $\alpha \neq 2$ and $k \neq 0$, in order to apply Theorem 5.1 it is necessary to have that $\left|\nabla_{x} \bar{y}\right| \in L^{\infty}\left(Q_{T}\right)$. Among the regularity results in this direction, we do not know any that can be used directly in our framework. Nevertheless, for some operators it is possible to combine the classical results of Ladyzhenskaya, Solonnikov and Ural'tseva [10] with a regularization of the state equation, the Ekeland's variational principle and Theorem 5.1 to establish the optimality conditions by means of a passage to the limit process. In particular, we will prove the following result

Theorem 5.2 Let be $\alpha \neq 2, k \neq 0$ and assume that

a) The operator $A$ is given by

$$
A y=-\operatorname{div}_{x}\left(a\left(\nabla_{x} y\right)\right)+a_{0}(y),
$$

where, in addition to (2.2)-(2.7), each coefficient $a_{j}(1 \leq j \leq n)$ possesses second order partial derivatives at almost every point of $\eta \in \mathbb{R}^{n}$ and satisfies

$$
\left|\frac{\partial^{2} a_{j}}{\partial \eta_{i} \partial \eta_{l}}(\eta)\right| \leq \Lambda_{2}(k+|\eta|)^{\alpha-3} \quad \text { a.e. } \eta \in \mathbb{R}^{n} \quad \forall 1 \leq i, l \leq n .
$$

b) Conditions (2.12) and (3.1)-(3.9) hold.

c) $\Gamma \in C^{2, \sigma}$, with $\sigma \in(0,1)$.

Then, given an optimal control $\bar{u}$ of $(P)$ and the corresponding optimal state $\bar{y}$, there exists an element $\bar{p} \in L^{\infty}\left(0, T ; L^{2}(\Omega)\right)$ satisfying (5.1), (5.2) and the following properties

$$
\begin{cases}\bar{p} \in L^{\alpha \wedge 2}\left(0, T ; W_{0}^{1, \alpha \wedge 2}(\Omega)\right), & \left(1+\left|\nabla_{x} \bar{y}\right|\right)^{\alpha-2} \nabla_{x} \bar{p} \in L^{\alpha^{\prime} \wedge 2}\left(Q_{T}\right)^{n}, \\ \bar{p}_{t} \in L^{\alpha^{\prime} \wedge 2}\left(0, T ; W^{-1, \alpha^{\prime} \wedge 2}(\Omega)\right), & \left(1+\left|\nabla_{x} \bar{y}\right|\right)^{\frac{\alpha-2}{2}} \nabla_{x} \bar{p} \in L^{2}\left(Q_{T}\right)^{n},\end{cases}
$$

where $\alpha \wedge 2=\min \{\alpha, 2\}$. Moreover, we have

$$
\begin{gathered}
\frac{1}{2}\|\bar{p}\|_{L^{\infty}\left(0, T ; L^{2}(\Omega)\right)}^{2}+\Lambda_{1} \int_{Q_{T}}\left(k+\left|\nabla_{x} \bar{y}\right|\right)^{\alpha-2}\left|\nabla_{x} \bar{p}\right|^{2} d x d t \leq \\
\leq \int_{Q_{T}}\left|\frac{\partial L}{\partial y}(x, t, \bar{y}, \bar{u}) \bar{p}\right| d x d t
\end{gathered}
$$


Let us point out that (in general) such an element $\bar{p}$ may be not unique.

We have chosen a particular type of operator to simplify the technicalities of the proof. A concrete example of an operator satisfying all the hypotheses imposed in Theorem 5.2 was given in Introduction (see (1.1)). At the end of the section, we will indicate a more general class of operators to which the previous result can be extended.

The next two subsections are devoted to the proof of Theorem 5.2.

\subsection{Approximating problems}

Let us begin by describing briefly the classical regularization procedure. For that purpose, we introduce the test function $\rho: \mathbb{R} \rightarrow[0,+\infty)$ given by

$$
\rho(s)= \begin{cases}\exp \left(\frac{1}{s^{2}-1}\right) & \text { if }|s|<1 \\ 0 & \text { if }|s| \geq 1\end{cases}
$$

For each natural number $m$ and positive real number $\epsilon$, let us define the functions

$$
\rho^{m}(\xi)=\frac{1}{c_{m}} \rho(|\xi|), \quad \rho_{\epsilon}^{m}(\xi)=\frac{1}{\epsilon^{m}} \rho^{m}\left(\frac{\xi}{\epsilon}\right) \quad \forall \xi \in \mathbb{R}^{m}
$$

where

$$
c_{m}=\int_{\mathbf{R}^{m}} \rho(|\xi|) d \xi .
$$

Next, given $\left(y_{0}, u\right) \in L^{\infty}(\Omega) \times L^{r}\left(Q_{T}\right)$ we will denote

$$
\tilde{y}_{0}(x)=\left\{\begin{array}{ll}
y_{0}(x) & \text { if } x \in \Omega \\
0 & \text { if } x \notin \Omega
\end{array} \quad \text { and } \quad \tilde{u}(x, t)= \begin{cases}u(x, t) & \text { if }(x, t) \in Q_{T} \\
0 & \text { if }(x, t) \notin Q_{T}\end{cases}\right.
$$

With these notations, let us define

$$
\begin{gathered}
a_{j}^{\epsilon}(\xi)=\left(\rho_{\epsilon}^{n} \star a_{j}\right)(\xi)-\left(\rho_{\epsilon}^{n} \star a_{j}\right)(0) \quad \forall 1 \leq j \leq n, \\
a_{0}^{\epsilon}(y)=\left(\rho_{\epsilon}^{1} \star a_{0}\right)(y)-\left(\rho_{\epsilon}^{1} \star a_{0}\right)(0),
\end{gathered}
$$

for all $y \in \mathbb{R}, \xi \in \mathbb{R}^{n}$, and

$$
\begin{gathered}
G^{\epsilon}[u](x, t)=\left(\rho_{\epsilon}^{n+1} \star \tilde{u}\right)(x, t) \cdot \phi_{\epsilon}(t) \\
G_{0}^{\epsilon}\left[y_{0}\right](x)=\left(\rho_{\epsilon}^{n} \star \tilde{y}_{0}\right)(x) \cdot \zeta_{\epsilon}(x)
\end{gathered}
$$

for all $(x, t) \in Q_{T}$, where $\star$ denotes the corresponding convolution product in each case,

$$
\begin{array}{llll}
\phi_{\epsilon} \in C_{0}^{\infty}(0, T), & 0 \leq \phi_{\epsilon}(t) \leq 1 & \text { and } \quad \phi_{\epsilon}(t) \rightarrow 1 & \text { as } \epsilon \rightarrow 0 \\
\zeta_{\epsilon} \in C_{0}^{\infty}(\Omega), & 0 \leq \zeta_{\epsilon}(x) \leq 1 & \text { and } \quad \zeta_{\epsilon}(x) \rightarrow 1 & \text { as } \epsilon \rightarrow 0 .
\end{array}
$$

It is well known that $a_{j}^{\epsilon} \in C^{\infty}\left(\mathbb{R}^{n}\right)$ for all $j \in\{1, \ldots, n\}, a_{0}^{\epsilon} \in C^{\infty}(\mathbb{R}), G^{\epsilon}[u] \in C^{\infty}\left(Q_{T}\right)$ and $G_{0}^{\epsilon}\left[y_{0}\right] \in C_{0}^{\infty}(\Omega)$. Furthermore, using the properties of the convolution product in a straightforward way, we can derive

Lemma 5.1 Let (2.2)-(2.7) and (5.7) hold with $k>0$. Then, for all $0<\epsilon<k$, we have

$$
\sum_{i, j=1}^{n} \frac{\partial a_{j}^{\epsilon}}{\partial \eta_{i}}(\eta) \xi_{i} \xi_{j} \geq \begin{cases}\Lambda_{1}(k-\epsilon+|\eta|)^{\alpha-2}|\xi|^{2} & \text { if } \alpha \geq 2 \\ \Lambda_{1}(k+\epsilon+|\eta|)^{\alpha-2}|\xi|^{2} & \text { if } \alpha \leq 2\end{cases}
$$




$$
\begin{gathered}
\sum_{i, j=1}^{n}\left|\frac{\partial a_{j}^{\epsilon}}{\partial \eta_{i}}(\eta)\right| \leq\left\{\begin{array}{cc}
\Lambda_{2}(k+\epsilon+|\eta|)^{\alpha-2} & \text { if } \alpha \geq 2 \\
\Lambda_{2}(k-\epsilon+|\eta|)^{\alpha-2} & \text { if } \alpha \leq 2
\end{array}\right. \\
\left|\frac{\partial^{2} a_{j}^{\epsilon}}{\partial \eta_{i} \partial \eta_{l}}(\eta)\right| \leq \begin{cases}\Lambda_{2}(k+\epsilon+|\eta|)^{\alpha-3} & \text { if } \alpha \geq 3 \\
\Lambda_{2}(k-\epsilon+|\eta|)^{\alpha-3} & \text { if } \alpha \leq 3\end{cases} \\
0 \leq \frac{d a_{0}^{\epsilon}}{d y}(y) \leq \varphi_{0}(|y|+k) \\
a_{0}^{\epsilon}(0)=a_{j}^{\epsilon}(0)=0
\end{gathered}
$$

for all $i, j, l \in\{1, \ldots, n\}, y \in \mathbb{R}$ and all $\eta, \xi \in \mathbb{R}^{n}$.

Next, let us introduce the following problem:

$$
\begin{cases}y_{t}-\operatorname{div}_{x}\left(a^{\epsilon}\left(\nabla_{x} y\right)\right)+a_{0}^{\epsilon}(y)=G^{\epsilon}[u] & \text { in } Q_{T} \\ y(x, t)=0 & \text { on } \Sigma \\ y(x, 0)=G_{0}^{\epsilon}\left[y_{0}\right](x) & \text { in } \Omega .\end{cases}
$$

Given $0<\epsilon<k / 2$ and $\left(y_{0}, u\right) \in L^{\infty}(\Omega) \times L^{r}\left(Q_{T}\right)$ with the exponent $r$ verifying (2.12), by virtue of Theorem 2.1, there exists a unique solution $y_{u}^{\epsilon}$ of problem (5.20) in the space $W^{\alpha}(0, T) \cap L^{\infty}\left(Q_{T}\right)$ (in fact, $y_{u}^{\epsilon}$ is a classical solution, see next subsection). We want to prove the convergence of $y_{u}^{\epsilon}$ towards $y_{u}$, the solution of (2.1). The following result actually gives us a little more.

Theorem 5.3 Let us assume (2.2)-(2.7), (2.12) and suppose that $\left\{u_{\epsilon}\right\}$ is a sequence in $L^{r}\left(Q_{T}\right)$ such that

$$
u_{\epsilon} \longrightarrow u \quad \text { weakly in } L^{r}\left(Q_{T}\right) \quad\left(\text { weakly }{ }^{\star} \text { if } r=+\infty\right) \quad \text { as } \epsilon \rightarrow 0
$$

Let us denote by $y_{\epsilon}$ the solution of (5.20) corresponding to $G^{\epsilon}\left[u_{\epsilon}\right]$. Then, it holds

$$
y_{\epsilon} \longrightarrow y_{u} \quad \text { in } L^{\alpha}\left(0, T ; W_{0}^{1, \alpha}(\Omega)\right) \cap C\left([0, T] ; L^{2}(\Omega)\right) \cap L^{q}\left(Q_{T}\right) \quad \text { as } \epsilon \rightarrow 0
$$

for all $q \in[1,+\infty)$, where $y_{u}$ is the solution of (2.1).

Proof. Thanks to the following classical estimates

$$
\left\|G^{\epsilon}\left[u_{\epsilon}\right]\right\|_{L^{r}\left(Q_{T}\right)} \leq\left\|u_{\epsilon}\right\|_{L^{r}\left(Q_{T}\right)} \leq C_{0}, \quad\left\|G_{0}^{\epsilon}\left[y_{0}\right]\right\|_{L^{\infty}(\Omega)} \leq\left\|y_{0}\right\|_{L^{\infty}(\Omega)} \quad \forall \epsilon \in(0, k / 2)
$$

and the conclusion of Theorem 2.1, there exists a constant $C_{1}$ independent of $\epsilon$ such that

$$
\left\|y_{\epsilon}\right\|_{W^{\alpha}(0, T)}+\left\|y_{\epsilon}\right\|_{L^{\infty}\left(Q_{T}\right)} \leq C_{1} \quad \forall \epsilon \in(0, k / 2)
$$

Recall Lemma 5.1 and notice that relations (5.15)-(5.17) can be written independently of $\epsilon$, when $\epsilon<k / 2$, by modifying the constants $\Lambda_{i}$ in a suitable form.

For $\alpha \geq 2$ the convergence (5.22) is a consequence of the following estimate:

$$
\begin{gathered}
\left\|y_{\epsilon}(t)-y_{u}(t)\right\|_{L^{2}(\Omega)}^{2}+\left\|y_{\epsilon}-y_{u}\right\|_{L^{\alpha}\left(0, T ; W_{0}^{1, \alpha}(\Omega)\right)}^{\alpha} \leq C_{2}\left(\int_{0}^{t}\left(\frac{\partial y_{\epsilon}}{\partial t}(s)-\frac{\partial y_{u}}{\partial t}(s), y_{\epsilon}(s)-y_{u}(s)\right) d s+\right. \\
+\int_{Q_{t}}\left(a\left(\nabla_{x} y_{\epsilon}\right)-a\left(\nabla_{x} y_{u}\right)\right)^{T}\left(\nabla_{x} y_{\epsilon}-\nabla_{x} y_{u}\right) d x d s+\left\|G_{0}^{\epsilon}\left[y_{0}\right]-y_{0}\right\|_{L^{2}(\Omega)}^{2}+
\end{gathered}
$$




$$
\begin{gathered}
\left.+\int_{Q_{t}}\left(a_{0}\left(y_{\epsilon}\right)-a_{0}\left(y_{u}\right)\right)\left(y_{\epsilon}-y_{u}\right) d x d s\right)= \\
=C_{2}\left(\int_{Q_{t}}\left(G^{\epsilon}\left[u_{\epsilon}\right]-u\right)\left(y_{\epsilon}-y_{u}\right) d x d s+\int_{Q_{t}}\left(a\left(\nabla_{x} y_{\epsilon}\right)-a^{\epsilon}\left(\nabla_{x} y_{\epsilon}\right)\right)^{T}\left(\nabla_{x} y_{\epsilon}-\nabla_{x} y_{u}\right) d x d s+\right. \\
\left.+\int_{Q_{t}}\left(a_{0}\left(y_{\epsilon}\right)-a_{0}^{\epsilon}\left(y_{\epsilon}\right)\right)\left(y_{\epsilon}-y_{u}\right) d x d s+\left\|G_{0}^{\epsilon}\left[y_{0}\right]-y_{0}\right\|_{L^{2}(\Omega)}^{2}\right)
\end{gathered}
$$

and therefore

$$
\begin{gathered}
\left\|y_{\epsilon}-y_{u}\right\|_{C\left([0, T] ; L^{2}(\Omega)\right)}^{2}+\left\|y_{\epsilon}-y_{u}\right\|_{L^{\alpha}\left(0, T ; W_{0}^{1, \alpha}(\Omega)\right)}^{\alpha} \leq \\
C_{3}\left(\left\|G^{\epsilon}\left[u_{\epsilon}\right]-u\right\|_{W^{\alpha}(0, T)^{\prime}}\left\|y_{\epsilon}-y_{u}\right\|_{W^{\alpha}(0, T)}\right. \\
+\sum_{j=1}^{n}\left\|a_{j}\left(\nabla_{x} y_{\epsilon}\right)-a_{j}^{\epsilon}\left(\nabla_{x} y_{\epsilon}\right)\right\|_{L^{\alpha^{\prime}\left(Q_{T}\right)}}\left\|y_{\epsilon}-y_{u}\right\|_{L^{\alpha}\left(Q_{T}\right)}+ \\
\left.+\left\|a_{0}\left(y_{\epsilon}\right)-a_{0}^{\epsilon}\left(y_{\epsilon}\right)\right\|_{L^{\alpha^{\prime}}\left(Q_{T}\right)}\left\|y_{\epsilon}-y_{u}\right\|_{L^{\alpha}\left(Q_{T}\right)}\right)+C_{2}\left\|G_{0}^{\epsilon}\left[y_{0}\right]-y_{0}\right\|_{L^{2}(\Omega)}^{2}
\end{gathered}
$$

The right-hand term goes to 0 as $\epsilon$ tends to 0 , thanks to (5.23), the convergences

$$
\begin{gathered}
a_{0}\left(y_{\epsilon}\right)-a_{0}^{\epsilon}\left(y_{\epsilon}\right) \longrightarrow 0 \quad \text { in } L^{\alpha^{\prime}}\left(Q_{T}\right) \\
a_{j}\left(\nabla_{x} y_{\epsilon}\right)-a_{j}^{\epsilon}\left(\nabla_{x} y_{\epsilon}\right) \longrightarrow 0 \quad \text { in } L^{\alpha^{\prime}}\left(Q_{T}\right) \quad \forall j \in\{1, \ldots, n\} \\
G_{0}^{\epsilon}\left[y_{0}\right] \longrightarrow y_{0} \quad \text { in } L^{2}(\Omega) \\
G^{\epsilon}\left[u_{\epsilon}\right] \longrightarrow u \quad \text { weakly in } L^{r}\left(Q_{T}\right)
\end{gathered}
$$

and the compact imbedding of $L^{r}\left(Q_{T}\right)$ into $W^{\alpha}(0, T)^{\prime}$, see $(2.17)$.

If $\alpha<2$, we obtain the same conclusion arguing once again as in (2.18).

Let us define the set

$$
\mathbb{K}_{1}=\left\{u \in \mathbb{K}:\|u-\bar{u}\|_{L^{r}\left(Q_{T}\right)} \leq 1\right\}
$$

where $\bar{u}$ is a fixed optimal control for $(P)$.

Next, we introduce the following family of approximating problems:

$$
\left(P_{\epsilon}\right) \min _{u \in \mathbf{K}_{1}} J_{\epsilon}(u)
$$

where the cost functional is given by

$$
J_{\epsilon}(u)=\int_{Q_{T}} L\left(x, t, y_{u}^{\epsilon}(x, t), u(x, t)\right) d x d t .
$$

The family of problems $\left(P_{\epsilon}\right)$ approximates to $(P)$ in the following sense:

Proposition 5.1 Let us suppose (2.2)-(2.7), (2.12) and (3.1)-(3.3). Then, it holds

$$
\lim _{\epsilon \rightarrow 0} \inf _{u \in \mathbf{K}_{1}} J_{\epsilon}(u)=\min _{u \in \mathbf{K}} J(u)=J(\bar{u})
$$


Proof. First of all, let us establish that

$$
m_{\epsilon} \equiv \inf _{u \in \mathbf{K}_{1}} J_{\epsilon}(u)>-\infty \quad \forall \epsilon \in(0, k / 2) .
$$

Thanks to the hypotheses (3.1)-(3.3), the boundedness of $\mathbb{K}_{1}$ and the conclusions of Theorem 2.1 and Lemma 5.1, we have that

$$
\begin{gathered}
\int_{Q_{T}} L\left(x, t, y_{u}^{\epsilon}, u\right) d x d t \geq \int_{Q_{T}} L(x, t, 0,0) d x d t+ \\
-\int_{Q_{T}}\left(h_{1}^{M}(x, t)+C_{M}|u|^{r / 2}\right)\left|y_{u}^{\epsilon}\right| d x d t-\int_{Q_{T}}\left(h_{0}^{M}(x, t)+C_{M}|u|^{r-1}\right)|u| d x d t \geq \\
\geq \int_{Q_{T}} L(x, t, 0,0) d x d t-\left(\left\|h_{1}^{M}\right\|_{L^{2}\left(Q_{T}\right)}+C_{M}\|u\|_{L^{r}\left(Q_{T}\right)}^{r / 2}\right)\left\|y_{u}^{\epsilon}\right\|_{L^{2}\left(Q_{T}\right)}+ \\
-\left(\left\|h_{0}^{M}\right\|_{L^{r^{\prime}\left(Q_{T}\right)}}+C_{M}\|u\|_{L^{r}\left(Q_{T}\right)}^{r-1}\right)\|u\|_{L^{r}\left(Q_{T}\right)} \geq \int_{Q_{T}} L(x, t, 0,0) d x d t+ \\
-\left(\left\|h_{1}^{M}\right\|_{L^{2}\left(Q_{T}\right)}+C_{M}\left(1+\|\bar{u}\|_{L^{r}\left(Q_{T}\right)}\right)^{r / 2}\right) M m\left(Q_{T}\right)+ \\
-\left(\left\|h_{0}^{M}\right\|_{L^{r^{\prime}}\left(Q_{T}\right)}+C_{M}\left(1+\|\bar{u}\|_{L^{r}\left(Q_{T}\right)}\right)^{r-1}\right)\left(1+\|\bar{u}\|_{L^{r}\left(Q_{T}\right)}\right) \quad \forall u \in \mathbb{K}_{1},
\end{gathered}
$$

where $M$ is a positive constant independent of $\epsilon$ and $u$ such that

$$
\left\|y_{u}^{\epsilon}\right\|_{L^{\infty}\left(Q_{T}\right)} \leq M \quad \forall \epsilon \in(0, k / 2) \quad \text { and } \quad \forall u \in \mathbb{K}_{1}
$$

Since $\bar{u} \in \mathbb{K}_{1}$, we see that

$$
m_{\epsilon} \leq J_{\epsilon}(\bar{u})=\int_{Q_{T}} L\left(x, t, y_{\bar{u}}^{\epsilon}, \bar{u}\right) d x d t
$$

By Theorem 5.3, we know that

$$
y_{\bar{u}}^{\epsilon} \longrightarrow \bar{y} \quad \text { in } L^{2}\left(Q_{T}\right) \text { and } \quad y_{\bar{u}}^{\epsilon}(x, t) \longrightarrow \bar{y}(x, t) \quad \text { a. e. }(x, t) \in Q_{T}
$$

at least for some subsequence.

Thus, using again the hypotheses (3.1)-(3.3) we deduce immediately that

$$
\limsup _{\epsilon \rightarrow 0} m_{\epsilon} \leq \lim _{\epsilon \rightarrow 0} J_{\epsilon}(\bar{u})=J(\bar{u}) .
$$

On the other hand, for any $\epsilon>0$ there exists $u_{\epsilon} \in \mathbb{K}_{1}$ such that

$$
J_{\epsilon}\left(u_{\epsilon}\right) \leq m_{\epsilon}+\epsilon
$$

Let us denote by $y_{\epsilon}$ the solution of $(5.20)$ corresponding to $G^{\epsilon}\left[u_{\epsilon}\right]$. Since $\mathbb{K}_{1}$ is bounded in $L^{r}\left(Q_{T}\right)$, we may assume that $u_{\epsilon}$ converges to some $\tilde{u}$ weakly in $L^{r}\left(Q_{T}\right)$ (weakly ${ }^{\star}$ if $r=+\infty$.) Hence, by Theorem 5.3, it follows again that

$$
y_{\epsilon} \longrightarrow y_{\tilde{u}} \quad \text { in } L^{2}\left(Q_{T}\right) \text { and } y_{\epsilon}(x, t) \longrightarrow y_{\tilde{u}}(x, t) \quad \text { a. e. }(x, t) \in Q_{T} .
$$

Now, let us consider $y_{u_{\epsilon}}$ the solution of (2.1) corresponding to $u_{\epsilon}$. By virtue of Theorem 2.1 and Corollary 2.1 we deduce once more that

$$
\left\|y_{u_{\epsilon}}\right\|_{L^{\infty}\left(Q_{T}\right)} \leq M \quad \forall \epsilon \quad \text { and } \quad y_{u_{\epsilon}}(x, t) \longrightarrow y_{\tilde{u}}(x, t) \quad \text { a. e. }(x, t) \in Q_{T}
$$


Combining these relations with the hypothesis (3.3), we derive that

$$
\begin{gathered}
m_{\epsilon}+\epsilon \geq J_{\epsilon}\left(u_{\epsilon}\right)=J\left(u_{\epsilon}\right)+\int_{Q_{T}}\left(L\left(x, t, y_{\epsilon}, u_{\epsilon}\right)-L\left(x, t, y_{u_{\epsilon}}, u_{\epsilon}\right)\right) d x d t \geq \\
\geq J(\bar{u})-\int_{Q_{T}}\left(h_{1}^{M}(x, t)+C_{M}\left|u_{\epsilon}\right|^{r / 2}\right)\left|y_{\epsilon}-y_{u_{\epsilon}}\right| d x d t \geq \\
\left.\geq J(\bar{u})-\left(\left\|h_{1}^{M}\right\|_{L^{2}\left(Q_{T}\right)}+C_{M}\left\|u_{\epsilon}\right\|_{L^{r}\left(Q_{T}\right)}^{r / 2}\right)\right)\left\|y_{\epsilon}-y_{u_{\epsilon}}\right\|_{L^{2}\left(Q_{T}\right)} .
\end{gathered}
$$

Making $\epsilon$ to tend to 0 , we conclude that

$$
J(\bar{u}) \leq \liminf _{\epsilon \rightarrow 0} m_{\epsilon}
$$

which, together with (5.31), gives (5.29).

Remark 5.1 In the proof of the previous proposition, we do not claim either that the problems $\left(P_{\epsilon}\right)$ have solutions or that the sequence $\left\{u_{\epsilon}\right\}$ converges to $\bar{u}$ in any sense. Actually, this might not be the case in general.

\subsection{Proof of Theorem 5.2}

First of all, let us point out that due to the smoothness of the functions $a^{\epsilon}$ it is possible to write out the equation of (5.20) in a developed form by expanding the divergence term. Moreover, thanks to the regularity of $G^{\epsilon}[u]$ and the compatibility condition between the boundary and the initial datum $G_{0}^{\epsilon}\left[y_{0}\right]$, we can apply [10, Theorem 4.1, pp. 558-559] and deduce that $y_{u}^{\epsilon} \in$ $C^{2+\sigma, 1+\sigma / 2}\left(\overline{Q_{T}}\right)$. The relevant fact here is that $\left|\nabla_{x} y_{u}^{\epsilon}\right| \in L^{\infty}\left(Q_{T}\right)$.

By Proposition 5.1 we know that

$$
0 \leq \delta_{\epsilon}^{2} \equiv J_{\epsilon}(\bar{u})-m_{\epsilon} \longrightarrow 0 \quad \text { as } \epsilon \rightarrow 0
$$

Therefore, by Ekeland's variational principle [7], for each $\epsilon>0$ we can find $u_{\epsilon} \in \mathbb{K}_{1}$ verifying the following properties

$$
\begin{gathered}
\left\|u_{\epsilon}-\bar{u}\right\|_{L^{r}\left(Q_{T}\right)} \leq \delta_{\epsilon} \\
J_{\epsilon}\left(u_{\epsilon}\right) \leq J_{\epsilon}(\bar{u}) \\
J_{\epsilon}(v)-J_{\epsilon}\left(u_{\epsilon}\right) \geq-\delta_{\epsilon}\left\|v-u_{\epsilon}\right\|_{L^{r}\left(Q_{T}\right) \quad \forall v \in \mathbb{K}_{1}}
\end{gathered}
$$

Last relation means that $u_{\epsilon}$ is an optimal control for the following problem

$$
\left(P_{\epsilon}^{\prime}\right) \min _{v \in \mathbf{K}_{1}}\left(J_{\epsilon}(v)+\delta_{\epsilon}\left\|v-u_{\epsilon}\right\|_{L^{r}\left(Q_{T}\right)}\right) .
$$

By virtue of the boundedness of the spatial gradient of $y_{u_{\epsilon}}^{\epsilon}$ in $Q_{T}$ and the convexity of the norm, it is possible to obtain the first order optimality system corresponding to $\left(P_{\epsilon}^{\prime}\right)$ for each $\epsilon \in(0, k / 2)$ as in Theorem 5.1, taking now into account that the necessary condition is

$$
J_{\epsilon}^{\prime}\left(u_{\epsilon}\right)\left(v-u_{\epsilon}\right)+\delta_{\epsilon}\left\|v-u_{\epsilon}\right\|_{L^{r}\left(Q_{T}\right)} \geq 0 \quad \forall v \in \mathbb{K}_{1} .
$$

Consequently, we obtain the following result for each $\epsilon \in(0, k / 2)$ : 
Theorem 5.4 Let us suppose that $\Gamma \in C^{2, \sigma}$ and assume the conditions (2.2)-(2.7), (2.12), (3.1)-(3.3) and (5.7) with $\alpha \neq 2$ and $k \neq 0$. Let $y_{\epsilon}$ be the state corresponding to $u_{\epsilon}$, i.e. $y_{\epsilon}=y_{u_{\epsilon}}^{\epsilon}$.

Then, there exists a unique element $p_{\epsilon} \in W^{2}(0, T)$ such that

$$
\begin{cases}-\frac{\partial p_{\epsilon}}{\partial t}-\operatorname{div}_{x}\left(\left[\frac{\partial a^{\epsilon}}{\partial \eta}\left(\nabla_{x} y_{\epsilon}\right)\right]^{T} \nabla_{x} p_{\epsilon}\right)+\frac{d a_{0}^{\epsilon}}{d y}\left(y_{\epsilon}\right) p_{\epsilon}=\frac{\partial L}{\partial y}\left(x, t, y_{\epsilon}, u_{\epsilon}\right) & \text { in } Q_{T} \\ p_{\epsilon}(x, t)=0 & \text { on } \Sigma, \\ p_{\epsilon}(x, T)=0 & \text { in } \Omega,\end{cases}
$$

and

$$
\int_{Q_{T}}\left(p_{\epsilon} G^{\epsilon}\left[v-u_{\epsilon}\right]+\frac{\partial L}{\partial u}\left(x, t, y_{\epsilon}, u_{\epsilon}\right)\left(v-u_{\epsilon}\right)\right) d x d t+\delta_{\epsilon}\left\|v-u_{\epsilon}\right\|_{L^{r}\left(Q_{T}\right)} \geq 0 \quad \forall v \in \mathbb{K}_{1}
$$

In order to pass to the limit in these optimality systems as $\epsilon \rightarrow 0$ it is necessary to distinguish the cases $\alpha>2$ and $\alpha<2$. As usual, we will describe in detail the first case and indicate the differences in the second one.

Taking into account the strong convergence of $u_{\epsilon}$ towards $\bar{u}$ in $L^{r}\left(Q_{T}\right)$, see (5.32) and (5.33), it follows from Theorem 5.3 that

$$
y_{\epsilon} \longrightarrow \bar{y} \quad \text { in } L^{\alpha}\left(0, T ; W_{0}^{1, \alpha}(\Omega)\right) \cap C\left([0, T] ; L^{2}(\Omega)\right) \cap L^{q}\left(Q_{T}\right) \quad \text { as } \epsilon \rightarrow 0
$$

for all $q \in[1,+\infty)$. Moreover, we know that $\left\{y_{\epsilon}\right\}$ is bounded in $L^{\infty}\left(Q_{T}\right)$, as it was claimed in (5.30).

Applying (5.36) to $p_{\epsilon}$, integrating in $(t, T)$ and using integration by parts, we obtain

$$
\begin{aligned}
& \frac{1}{2}\left\|p_{\epsilon}(t)\right\|_{L^{2}(\Omega)}^{2}+\int_{Q^{t}}\left(\nabla_{x} p_{\epsilon}\right)^{T} \frac{\partial a^{\epsilon}}{\partial \eta}\left(\nabla_{x} y_{\epsilon}\right) \nabla_{x} p_{\epsilon} d x d s+ \\
& +\int_{Q^{t}} \frac{d a_{0}^{\epsilon}}{d y}\left(y_{\epsilon}\right)\left|p_{\epsilon}\right|^{2} d x d s=\int_{Q^{t}} \frac{\partial L}{\partial y}\left(x, s, y_{\epsilon}, u_{\epsilon}\right) p_{\epsilon} d x d s
\end{aligned}
$$

for each $t \in[0, T)$, where $Q^{t}=\Omega \times(t, T)$.

Together with (3.3), (5.15) and (5.18), previous estimate with $t=0$ implies (remember that we are assuming $\alpha>2$ )

$$
\begin{gathered}
\Lambda_{1} k_{1}^{\alpha-2}\left\|p_{\epsilon}\right\|_{L^{2}\left(0, T ; H_{0}^{1}(\Omega)\right)}^{2} \leq \int_{Q_{T}}\left(\nabla_{x} p_{\epsilon}\right)^{T} \frac{\partial a^{\epsilon}}{\partial \eta}\left(\nabla_{x} y_{\epsilon}\right) \nabla_{x} p_{\epsilon} d x d s \leq \\
\leq C_{1}\left\|p_{\epsilon}\right\|_{L^{2}\left(0, T ; H_{0}^{1}(\Omega)\right)},
\end{gathered}
$$

with $k_{1}=\min \{k / 2,1\}$ for each $\epsilon<k / 2$. Hence, we get the boundedness of the sequence $\left\{p_{\epsilon}\right\}$ in $L^{2}\left(0, T ; H_{0}^{1}(\Omega)\right) \cap L^{\infty}\left(0, T ; L^{2}(\Omega)\right)$.

Furthermore, it follows from (5.15) and (5.40) that $\left\{\left(k-\epsilon+\left|\nabla_{x} y_{\epsilon}\right|\right)^{(\alpha-2) / 2} \nabla_{x} p_{\epsilon}\right\}$ is bounded in $L^{2}\left(Q_{T}\right)^{n}$.

Finally, by virtue of Young's inequality we obtain that

$$
\begin{gathered}
\left\|\left(k-\epsilon+\left|\nabla_{x} y_{\epsilon}\right|\right)^{\alpha-2} \nabla_{x} p_{\epsilon}\right\|_{L^{\alpha^{\prime}}\left(Q_{T}\right)^{n}}^{\alpha^{\prime}} \leq \\
\leq C_{2}\left(\left\|k-\epsilon+\left|\nabla_{x} y_{\epsilon}\right|\right\|_{L^{\alpha}\left(Q_{T}\right)}^{\alpha}+\left\|\left(k-\epsilon+\left|\nabla_{x} y_{\epsilon}\right|\right)^{(\alpha-2) / 2} \nabla_{x} p_{\epsilon}\right\|_{L^{2}\left(Q_{T}\right)^{n}}^{2}\right) \leq C_{3}
\end{gathered}
$$


Thus, with the aid of (5.38), we can easily deduce the existence of an element $\bar{p}$ such that

$$
\begin{gathered}
p_{\epsilon} \longrightarrow \bar{p} \quad \text { weakly in } L^{2}\left(0, T ; H_{0}^{1}(\Omega)\right) \\
p_{\epsilon} \longrightarrow \bar{p} \quad \text { weakly }^{\star} \text { in } L^{\infty}\left(0, T ; L^{2}(\Omega)\right) \\
\left(k-\epsilon+\left|\nabla_{x} y_{\epsilon}\right|\right)^{(\alpha-2) / 2} \nabla_{x} p_{\epsilon} \longrightarrow\left(k+\left|\nabla_{x} \bar{y}\right|\right)^{(\alpha-2) / 2} \nabla_{x} \bar{p} \quad \text { weakly in } L^{2}\left(Q_{T}\right)^{n} \\
\left(k-\epsilon+\left|\nabla_{x} y_{\epsilon}\right|\right)^{\alpha-2} \nabla_{x} p_{\epsilon} \longrightarrow\left(k+\left|\nabla_{x} \bar{y}\right|\right)^{\alpha-2} \nabla_{x} \bar{p} \quad \text { weakly in } L^{\alpha^{\prime}}\left(Q_{T}\right)^{n}
\end{gathered}
$$

Now we can pass to the limit in (5.37) without any difficulty to obtain

$$
\int_{Q_{T}}\left(\bar{p}+\frac{\partial L}{\partial u}(x, t, \bar{y}, \bar{u})\right)(v-\bar{u}) d x d t \geq 0 \quad \forall v \in \mathbb{K}_{1}
$$

Given any $u \in \mathbb{K}$ let us pick $\tau>0$ such that $v=\bar{u}+\tau(u-\bar{u}) \in \mathbb{K}_{1}$. If we take such a $v$ in (5.45) we prove (5.2).

On the other hand, arguing in a straightforward way we have

$$
\begin{array}{cc}
\frac{d a_{0}^{\epsilon}}{d y}\left(y_{\epsilon}\right) \longrightarrow \frac{d a_{0}}{d y}(\bar{y}) \quad \text { in } L^{q}\left(Q_{T}\right) & \forall q \in[1,+\infty) \\
\frac{\partial a^{\epsilon}}{\partial \eta}\left(\nabla_{x} y_{\epsilon}(x, t)\right) \longrightarrow \frac{\partial a}{\partial \eta}\left(\nabla_{x} \bar{y}(x, t)\right) & \text { a. e. }(x, t) \in Q_{T} \\
\frac{\partial L}{\partial y}\left(\cdot, \cdot, y_{\epsilon}, u_{\epsilon}\right) \longrightarrow \frac{\partial L}{\partial y}(\cdot, \cdot, \bar{y}, \bar{u}) & \text { in } L^{2}\left(Q_{T}\right)
\end{array}
$$

Hence, combining (5.16) with (5.44) and (5.47) we get

$$
\left[\frac{\partial a^{\epsilon}}{\partial \eta}\left(\nabla_{x} y_{\epsilon}\right)\right]^{T} \nabla_{x} p_{\epsilon} \longrightarrow\left[\frac{\partial a}{\partial \eta}\left(\nabla_{x} \bar{y}\right)\right]^{T} \nabla_{x} \bar{p} \quad \text { weakly in } L^{\alpha^{\prime}}\left(Q_{T}\right)^{n}
$$

Thanks to previous convergences, we can apply (5.36) to any element $\psi \in C_{0}^{\infty}\left(Q_{T}\right)$ and make $\epsilon$ to tend to 0 , deriving that

$$
-\bar{p}_{t}-\operatorname{div}_{x}\left\{\left[\frac{\partial a}{\partial \eta}\left(\nabla_{x} \bar{y}\right)\right]^{T} \nabla_{x} \bar{p}\right\}+\frac{d a_{0}}{d y}(\bar{y}) \bar{p}=\frac{\partial L}{\partial y}(x, t, \bar{y}, \bar{u}) \quad \text { in } \quad Q_{T}
$$

which in particular implies that $\bar{p}_{t} \in L^{\alpha^{\prime}}\left(0, T ; W^{-1, \alpha^{\prime}}(\Omega)\right)$.

Actually, we have proved that

$$
\frac{\partial p_{\epsilon}}{\partial t} \longrightarrow \frac{\partial \bar{p}}{\partial t} \quad \text { weakly in } \quad L^{\alpha^{\prime}}\left(0, T ; W^{-1, \alpha^{\prime}}(\Omega)\right),
$$

which together with (5.41) implies that

$$
p_{\epsilon} \longrightarrow \bar{p} \quad \text { weakly in } W^{1, \alpha^{\prime}}\left(0, T ; W^{-1, \alpha^{\prime}}(\Omega)\right)
$$

By virtue of the imbedding (2.8) we deduce

$$
p_{\epsilon} \longrightarrow \bar{p} \quad \text { weakly in } C\left([0, T] ; W^{-1, \alpha^{\prime}}(\Omega)\right)
$$

and therefore

$$
0=p_{\epsilon}(\cdot, T) \longrightarrow \bar{p}(\cdot, T) \quad \text { weakly in } W^{-1, \alpha^{\prime}}(\Omega)
$$

So, $\bar{p}(\cdot, T)=0$.

To complete the proof when $\alpha>2$ it is enough to take into account that inequality (5.9) is a simple consequence of some previous convergences, (5.15), (5.18) and (5.39).

In the case $\alpha<2$ and $k \neq 0$ the argumentation changes in the following points: 
- The sequence $\left\{p_{\epsilon}\right\}$ is bounded in $L^{2}\left(Q_{T}\right)$ as a direct consequence of (5.39) and the continuous imbedding of $L^{\infty}\left(0, T ; L^{2}(\Omega)\right)$ in $L^{2}\left(Q_{T}\right)$

- The boundedness of $\left\{p_{\epsilon}\right\}$ in $L^{\alpha}\left(0, T ; W_{0}^{1, \alpha}(\Omega)\right)$ can be obtained now using again (5.15), (5.38), (5.39) and the Hölder's inequality as in (4.24)

- The sequence $\left\{\left(k-\epsilon+\left|\nabla_{x} y_{\epsilon}\right|\right)^{\alpha-2} \nabla_{x} p_{\epsilon}\right\}$ is bounded in $L^{2}\left(Q_{T}\right)^{n}$, because here

$$
\left\|\left(k-\epsilon+\left|\nabla_{x} y_{\epsilon}\right|\right)^{\alpha-2} \nabla_{x} p_{\epsilon}\right\|_{L^{2}\left(Q_{T}\right)^{n}} \leq C_{4}\left\|\left(k-\epsilon+\left|\nabla_{x} y_{\epsilon}\right|\right)^{(\alpha-2) / 2} \nabla_{x} p_{\epsilon}\right\|_{L^{2}\left(Q_{T}\right)^{n}} \leq C_{5}
$$

and therefore, we derive

$$
\left[\frac{\partial a^{\epsilon}}{\partial \eta}\left(\nabla_{x} y_{\epsilon}\right)\right]^{T} \nabla_{x} p_{\epsilon} \longrightarrow\left[\frac{\partial a}{\partial \eta}\left(\nabla_{x} \bar{y}\right)\right]^{T} \nabla_{x} \bar{p} \quad \text { weakly in } L^{2}\left(Q_{T}\right)^{n}
$$

for some limit point $\bar{p}$ of $\left\{p_{\epsilon}\right\}$ in $L^{\alpha}\left(0, T ; W_{0}^{1, \alpha}(\Omega)\right) \cap L^{\infty}\left(0, T ; L^{2}(\Omega)\right)$ with the weak (resp. weak $^{\star}$ ) topology

- Finally, it is easily verified that $\bar{p}$ satisfies (5.50); moreover, we have

$$
p_{\epsilon} \longrightarrow \bar{p} \quad \text { weakly in } W^{1, \alpha}\left(0, T ; H^{-1}(\Omega)\right) \subset C\left([0, T] ; H^{-1}(\Omega)\right),
$$

from which we obtain that $\bar{p}(\cdot, T)=0$

\subsection{Generalizations}

It can be showed that Theorem 5.2 continues to hold for operators

$$
y_{t}-\operatorname{div}_{x}\left(a\left(x, t, \nabla_{x} y\right)\right)+a_{0}(x, t, y)
$$

verifying together with $(2.2)-(2.7)$ that $\frac{\partial a_{0}}{\partial y} \in C\left(Q_{T} \times \mathbb{R}\right)$,

$$
\left|a_{j}(x, t, \eta)-a_{j}\left(x^{\prime}, t^{\prime}, \eta\right)\right| \leq \Lambda_{2}(1+|\eta|)^{\alpha-1}\left(\left|x-x^{\prime}\right|^{\sigma}+\left|t-t^{\prime}\right|^{\sigma}\right) \quad j=1, \ldots, n
$$

for all $x, x^{\prime} \in \Omega, t, t^{\prime} \in(0, T)$ and $\eta \in \mathbb{R}^{n}$, with $\sigma \in(0,1)$; furthermore it is necessary to suppose that each coefficient $a_{j}$ possesses second order partial derivatives with respect to $x$ and $\eta$ at almost every point of $Q_{T} \times R^{n}$, satisfying

$$
\begin{aligned}
\left|\frac{\partial a_{j}}{\partial x_{i}}(x, t, \eta)\right| & \leq \Lambda_{2}(k+|\eta|)^{\alpha} \\
\left|\frac{\partial^{2} a_{j}}{\partial \eta_{i} \partial \eta_{l}}(x, t, \eta)\right| & \leq \Lambda_{2}(k+|\eta|)^{\alpha-3} \\
\left|\frac{\partial^{2} a_{j}}{\partial x_{i} \partial \eta_{l}}(x, t, \eta)\right| & \leq \Lambda_{2}(k+|\eta|)^{\alpha-1} \\
\left|\frac{\partial^{2} a_{j}}{\partial x_{i} \partial x_{l}}(x, t, \eta)\right| & \leq \Lambda_{2}(k+|\eta|)^{\alpha+1}
\end{aligned}
$$

for all $1 \leq i, j, l \leq n$ and a. e. $(x, t, \eta) \in Q_{T} \times \mathbb{R}^{n}$. 
The proof uses essentially the same arguments as before, the main difference being the definition of the coefficients $a_{0}^{\epsilon}$ and $a_{j}^{\epsilon}$. In order to make possible the convolution products with the approximation of the identity $\left\{\rho_{\epsilon}\right\}$, we must extend the domain of definition of the coefficients $a_{0}$ and $a_{j}$ to $R^{n+2}$ and $R^{2 n+1}$ respectively. These extensions (that we denote by $\tilde{a}_{j}$ ) can be constructed in a standard way (see, for instance, [10]). Moreover, this construction preserves the conditions satisfied by each function, i.e. each $\tilde{a}_{j}$ verifies the same conditions than the corresponding $a_{j}$, for all $0 \leq j \leq n$ in a domain $\tilde{Q}_{T} \times R^{n}$, where $\tilde{Q}_{T}$ is an open subset of $\mathbb{R}^{n+1}$ such that $\overline{Q_{T}} \subset \tilde{Q}_{T}$.

With these notations, we define

$$
a_{j}^{\epsilon}(x, t, \xi)=\left(\rho_{\epsilon}^{2 n+1} \star \tilde{a}_{j}\right)(x, t, \xi)-\left(\rho_{\epsilon}^{2 n+1} \star \tilde{a}_{j}\right)(x, t, 0) \quad \forall 1 \leq j \leq n,
$$

and

$$
a_{0}^{\epsilon}(x, t, y)=\left(\rho_{\epsilon}^{n+2} \star \tilde{a}_{0}\right)(x, t, y)-\left(\rho_{\epsilon}^{n+2} \star \tilde{a}_{0}\right)(x, t, 0),
$$

that still satisfy the same conditions than $a_{j}$ and $a_{0}$ for all $\epsilon \in\left(0, \epsilon_{0}\right)$, with

$$
\epsilon_{0}=\min \left\{k / 2, d\left(Q_{T}, \mathbb{R}^{n+1} \backslash \tilde{Q}_{T}\right)\right\} .
$$

Let us point out that the additional assumptions (5.52)-(5.55) allow us to apply [10, Theorem 4.1, pp. 558-559] to the regularized problems.

Finally, let us notice that the following operator

$$
y_{t}(x, t)-\sum_{j=1}^{n} \frac{\partial}{\partial x_{j}}\left(\left(k+\left|\frac{\partial y}{\partial x_{j}}(x, t)\right|\right)^{\alpha-2} \frac{\partial y}{\partial x_{j}}(x, t)\right)
$$

does not enter into the framework of this paper, because of hypothesis (2.4). However the same type of results can be obtained for optimal control problems governed by this equation, by changing slightly the argumentation of the proofs; see also [17].

\section{References}

[1] N. U. Ahmed. Optimal control of a class of strongly nonlinear parabolic systems. J. of Math. Anal. \& Appl., 61:188-207, 1977.

[2] V. Barbu. Optimal control of variational inequalities. Pitman, Boston-London-Melbourne, 1984 .

[3] E. Casas and L.A. Fernández. Optimal control of quasilinear elliptic equations with non differentiable coefficients at the origin. Revista Matemática de la Universidad Complutense de Madrid, 4(2-3):227-250, 1991.

[4] E. Casas and L.A. Fernández. Distributed control of systems governed by a general class of quasilinear elliptic equations. J. of Differential Equations, 1993.

[5] E. Casas and J. Yong. Maximum principle for state-constrained optimal control problems governed by quasilinear elliptic equations. Technical Report 1063, IMA, November 1992.

[6] I. Chryssoverghi. Nonconvex optimal control of nonlinear monotone parabolic systems. Systems 8 Control Letters, 8:55-62, 1986. 
[7] I. Ekeland. Nonconvex minimization problems. Bull. Amer. Math. Soc., 1(3):76-91, 1979.

[8] I. Ekeland and R. Temam. Analyse Convexe et Problèmes Variationnels. Dunod-Gauthier Villars, Paris, 1974.

[9] H. O. Fattorini. Optimal control problems for distributed parameter systems governed by semilinear parabolic equations in $L^{1}$ and $L^{\infty}$ spaces. In K.H. Hoffmann and W. Krabs, editors, Optimal Control of Partial Differential Equations, pages 68-80, Springer-Verlag, Berlin-Heidelberg-New York, 1991. Lecture Notes in Control and Information Sciences 149.

[10] O.A. Ladyzhenskaya, V.A. Solonnikov, and N.N. Ural'tseva. Linear and Quasilinear Equations of Parabolic Type. Am. Math. Soc, Providence, R. I., 1968.

[11] J. L. Lions. Optimisation pour certaines classes d'équations d'évolution non linéaires. Ann. Mat. Pura Appl., 72:275-293, 1966.

[12] J.L. Lions. Quelques Méthodes de Résolution des Problèmes aux Limites non Linéaires. Dunod, Paris, 1969.

[13] S. Nabadan and E. S. Noussair. Existence theorems for optimal control of quasilinear parabolic partial differential equations. J. Austral. Math. Soc., 21:90-101, 1979.

[14] E. S. Noussair, S. Nabadan, and K. L. Teo. On the existence of optimal controls for quasilinear parabolic partial differential equations. J. Optimization Theory \& Appl., 34(1):99-115, 1981.

[15] L. Pan and J. Yong. Optimal control for quasilinear retarded parabolic systems. Math. Systems, Estimation \& Control, to appear.

[16] N. S. Papageorgiou. On the optimal control of strongly nonlinear evolution equations. $J$. of Math. Anal. \& Appl., 164:83-103, 1992.

[17] D. Tiba. Optimality conditions for distributed control problems with nonlinear state equation. SIAM J. on Control \& Optimiz., 23(1):85-110, 1985.

[18] D. Tiba. Optimal control of nonsmoooth distributed parameter systems. Springer-Verlag, Berlin-Heidelberg-New York, 1990. Lecture Notes in Mathematics 1459.

[19] F. Tröltzsch. Optimality Conditions for Parabolic Control Problems and Applications. Teubner-Texte, Leipzig, 1984. 